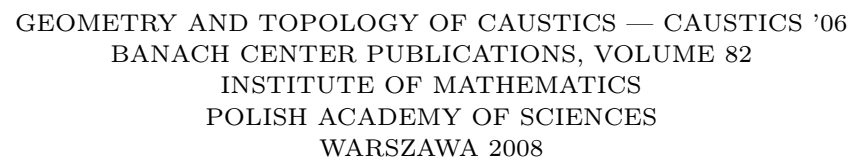

\title{
PROJECTIONS OF SURFACES WITH SINGULAR BOUNDARY
}

\author{
HASSAN BABIKER \\ Faculty of Mathematics and Information Science, Warsaw University of Technology \\ Plac Politechniki 1, 00-661 Warszawa, Poland \\ E-mail:babiker@wp.pl
}

\begin{abstract}
Singular projections of generic 2-dim surfaces in $\mathbb{R}^{3}$ with singular boundary to 2 -space are studied. The case of projections of surfaces with nonsingular boundary has been treated by Bruce and Giblin. The aim of this paper is to generalise these results to the simplest singular case where the boundary of the surface consists of two transversally intersecting lines. Local models for germs of generic singular projections of corank $\leq 1$ and codimension $\leq 3$ are given. We also present geometrical realisations via the notion of symmetrical unfolding.
\end{abstract}

Introduction. Projections of surfaces arise in many mathematical problems. A typical example is analysis of an apparent contour of a generic smooth 2-dimensional surface. Singularities of projections of surfaces have been studied by many authors. The classification of projections of smooth compact 2-surfaces in $\mathbb{R}^{3}$ onto $\mathbb{R}^{2}$ has been given by Arnold in [1].

The problem of classification of projections of surfaces with smooth boundary, as was shown in work by Bruce and Giblin in [2] may be reduced to the classification of map-germs $\left(\mathbb{R}^{2}, 0\right) \rightarrow\left(\mathbb{R}^{2}, 0\right)$ with respect to the subgroup of diffeomorphisms preserving a distinguished line in the source and the full group of diffeomorphisms in the target. It turns out that this group of equivalences is a geometric subgroup of $\mathcal{A}$ in the sense of Damon (see [6]). Using generalised determinacy theorems for geometric subgroups and the standard construction of the universal deformations Bruce and Giblin have given a complete list of local models of projections of 2-dim surfaces with smooth boundary.

Our goal is to study projections of surfaces with singular boundary. Here we make a first step and consider a surface with boundary which consists of two transversally

2000 Mathematics Subject Classification: Primary 58K40; Secondary 58K20.

Key words and phrases: projections, surfaces, classifications, normal forms.

The author would like to thank Prof. Stanisław Janeczko for his supervision and precious advice.

The paper is in final form and no version of it will be published elsewhere. 
intersecting lines. In section 1 we give notation and recall basic definitions and constructions. In section 2 we state the problem for surfaces with singular boundary, introduce the algebraic structure of corresponding equivalence group and tangent spaces. Moreover, the proofs of required determinacy theorems and complete transversal theorem are given. An inductive classification on the jet level up to codimension 3 is presented in section 3 . Finally section 4 is devoted to presentation of classification results and the list of universal unfoldings. We also give geometric realizations for versal unfoldings of map-germs in section 5 .

1. Preliminaries on singularities of smooth mappings. Let us begin with notation and recalling some of the basic definitions in the theory of singularities of smooth mappings.

Definition 1.1. A germ at $0 \in \mathbb{R}^{n}$ of smooth mapping $\left(\mathbb{R}^{n}, 0\right) \rightarrow \mathbb{R}^{p}$ is an equivalence class in the space of smooth mappings $C^{\infty}\left(\mathbb{R}^{n}, \mathbb{R}^{p}\right)$ of the following equivalence relation: $\left(\forall f, g \in C^{\infty}\left(\mathbb{R}^{n}, \mathbb{R}^{p}\right)\right) f \sim g \Leftrightarrow$ there exists an open neighbourhood $\mathcal{O}$ of $0 \in \mathbb{R}^{n}$ such that $\left.\left.f\right|_{\mathcal{O}}=\left.g\right|_{\mathcal{O}}\right\}$.

The space of smooth map-germs and its algebraic structure. By $\mathcal{E}_{n, p}$ we denote the vector space of smooth map-germs $\left(\mathbb{R}^{n}, 0\right) \rightarrow \mathbb{R}^{p} . \mathcal{E}_{n, 1}=\mathcal{E}_{n}$ has the structure of a local $\mathbb{R}$-algebra with unique maximal ideal $\mathbf{m}_{n} \subset \mathcal{E}_{n}$, where $\mathbf{m}_{n}=\left\{f \in \mathcal{E}_{n} \mid f(0)=0\right\}$. The space $\mathcal{E}_{n, p}$ has the structure of a free $\mathcal{E}_{n}$-module coming from the following canonical identification: $\mathcal{E}_{n, p}=\underbrace{\mathcal{E}_{n, 1} \oplus \ldots \oplus \mathcal{E}_{n, 1}}_{p}$.

$\mathcal{A}$-equivalence of map-germs. From now on we will assume that all germs preserve zero, which means: $f \in \mathbf{m}_{n} \cdot \mathcal{E}_{n, p}$. Let $\mathcal{D}(n)$ denote the group of germs of diffeomorphisms $\left(\mathbb{R}^{n}, 0\right) \rightarrow\left(\mathbb{R}^{n}, 0\right)$.

Definition 1.2. Two map-germs $f, g \in \mathbf{m}_{n} \cdot \mathcal{E}_{n, p}$ are $\mathcal{A}$-equivalent if there exist germs of diffeomorphisms $\psi \in \mathcal{D}(p)$ and $\varphi \in \mathcal{D}(n)$ such that:

$$
g=\psi \circ f \circ \varphi^{-1} \text {. }
$$

$\mathcal{A}$-equivalence is denoted by $f \sim_{\mathcal{A}} g$.

The action of the group $\mathcal{A}$ on the space $\mathcal{E}_{n, p}$. Let the product group $\mathcal{A}=\mathcal{D}(n) \times \mathcal{D}(p)$ act on the space $\mathbf{m}_{n} \cdot \mathcal{E}_{n, p}$ as follows

$$
\forall(\varphi, \psi) \in \mathcal{D}(n) \times \mathcal{D}(p) \forall f \in \mathbf{m}_{n} \cdot \mathcal{E}_{n, p}(\varphi, \psi) . f=\psi \circ f \circ \varphi^{-1} .
$$

The orbit of the germ $f \in \mathbf{m}_{n} \cdot \mathcal{E}_{n, p}$ in the above action is denoted by $\mathcal{A}$. $f$. Two map-germs are $\mathcal{A}$-equivalent iff they lie in the same $\mathcal{A}$-orbit.

Jets of map-germs and their orbits

Definition 1.3. The vector space of $k$-jets of map-germs is the quotient space:

$$
J_{n, p}^{k}=\mathbf{m}_{n} \cdot \mathcal{E}_{n, p} / \mathbf{m}_{n}^{k+1} \cdot \mathcal{E}_{n, p} .
$$

REMARK 1.1. A $k$-jet of the map-germ $f \in \mathbf{m}_{n} \cdot \mathcal{E}_{n, p}$ is identified with its Taylor series expansion at $0 \in \mathbb{R}^{n}$ up to order $k$. 
Definition 1.4. The canonical projection onto the space of $k$-jets is denoted by

$$
j^{k}: \mathbf{m}_{n} \cdot \mathcal{E}_{n, p} \rightarrow J_{n, p}^{k} .
$$

The space of $k$-jets may be identified with

$$
J_{n, p}^{k} \cong \underset{i=1}{p} B_{n}^{k}
$$

where $B_{n}^{k}$ denotes the vector space of polynomials in $n$ variables of degree $\leq k$.

The sequence of powers of the maximal ideal in $\mathcal{E}_{n}$ defines a filtration of $\mathcal{E}_{n, p}$ such that

$$
\mathcal{E}_{n, p} \supset \mathbf{m}_{n} \cdot \mathcal{E}_{n, p} \supset \mathbf{m}_{n}^{2} \cdot \mathcal{E}_{n, p} \supset \ldots
$$

We also introduce the space of homogeneous mappings of degree $k$ :

$$
H_{n, p}^{k}=\mathbf{m}_{n}^{k} \cdot \mathcal{E}_{n, p} / \mathbf{m}_{n}^{k+1} \cdot \mathcal{E}_{n, p} .
$$

Action of the group of $k$-jets of diffeomorphisms on the space of $k$-jets. Let $\mathcal{A}_{r}$ denote the subset of those diffeomorphisms in $\mathcal{A}$, for which $r$-jets are identity. As the $r$-jet of the composition of two mappings depends only on the $r$-jets of its components it follows that $\mathcal{A}_{r}$ is a normal subgroup of $\mathcal{A}$. Moreover $\mathcal{A}_{r}$ acts trivially on $J_{n, p}^{r}$. This in turn gives us a well defined quotient action of $J^{r} \mathcal{A}$ on the space $J_{n, p}^{r}$ where

$$
\mathcal{A} / \mathcal{A}_{r}=J^{r} \mathcal{A} \text {. }
$$

The group of germs of diffeomorphisms $\left(\mathbb{R}^{n}, 0\right) \rightarrow\left(\mathbb{R}^{n}, 0\right)$ does not have a manifold structure, hence is not a Lie group. However the finite dimensional approximations by $k$-jets of diffeomorphisms denoted as $J^{k} \mathcal{A}$ form an open subset in the space $J_{n, n}^{k}$ and so admit a finite dimensional differentiable structure. The composition defined by

$$
\left(j^{k} f\right) *\left(j^{k} g\right)=j^{k}(f \circ g)
$$

together with the differentiable structure induced from $J_{n, n}^{k}$ provides a Lie group structure.

Definition 1.5. The orbit of the $k$-jet $j^{k} f$ of the map-germ $f:\left(\mathbb{R}^{n}, 0\right) \rightarrow\left(\mathbb{R}^{n}, 0\right)$ is the orbit of the element $j^{k} f$ under the action of $J^{k} \mathcal{A}$.

The notion of $k$-determinancy and sufficiency

Definition 1.6. Let $f:\left(\mathbb{R}^{n}, 0\right) \rightarrow \mathbb{R}^{p}$ be a map-germ. We call $f k$-A-determined iff for all $g \in \mathcal{E}_{n, p}$ we have

$$
j^{k} f=j^{k} g \Rightarrow f \sim_{\mathcal{A}} g .
$$

Definition 1.7. The smallest such $k \in \mathbb{N}$ such that $f \in \mathcal{E}_{n, p}$ is $k$-A-determined is called the determinacy degree.

Definition 1.8. A $k$-jet $g \in J_{n, p}^{k}$ is called a sufficient jet iff $g$ is $k$-determined.

Tangent space to the orbit of a map-germ. Let $f:\left(\mathbb{R}^{n}, 0\right) \rightarrow\left(\mathbb{R}^{p}, 0\right)$ be a germ of smooth mapping.

Definition 1.9. A germ of smooth vector field along $f$ is a map-germ $\xi:\left(\mathbb{R}^{n}, 0\right) \rightarrow T \mathbb{R}^{p}$ such that $\pi_{p} \circ \xi=f$ where $\pi_{p}: T \mathbb{R}^{p} \rightarrow \mathbb{R}^{p}$ denotes the canonical projection of the tangent bundle. 
In what follows we will use the notation

- $V\left(\mathbb{R}^{n}\right)$, the $\mathcal{E}_{n}$-module of germs at $0 \in \mathbb{R}^{n}$ of smooth vector fields on $\mathbb{R}^{n}$,

- $V(f)$, the $\mathcal{E}_{n}$-module of germs at $0 \in \mathbb{R}^{n}$ of smooth vector fields along $f$.

The mapping $f$ induces the natural transformations

$$
\begin{array}{cll}
t f: V\left(\mathbb{R}^{n}\right) \rightarrow V(f), & t f(X)=d f(X) & \text { for } X \in V\left(\mathbb{R}^{n}\right), \\
w f: V\left(\mathbb{R}^{p}\right) \rightarrow V(f), & w f(Y)=Y \circ f & \text { for } Y \in V\left(\mathbb{R}^{p}\right) .
\end{array}
$$

REMARK 1.2. Under the canonical identification of $T \mathbb{R}^{p} \cong \mathbb{R}^{p} \times \mathbb{R}^{p}$ the space $V(f)$ may be identified with $\mathcal{E}_{n, p}$.

Definition 1.10. The tangent space to the map-germ $f$ is defined to be

$$
T f=t f\left(V\left(\mathbb{R}^{n}\right)\right)+w f\left(V\left(\mathbb{R}^{p}\right)\right) .
$$

Definition 1.11. The tangent space to the $\mathcal{A}$-orbit of the map-germ is defined to be

$$
T \mathcal{A} . f=t f\left(\mathbf{m}_{n} \cdot V\left(\mathbb{R}^{n}\right)\right)+w f\left(\mathbf{m}_{p} \cdot V\left(\mathbb{R}^{p}\right)\right) .
$$

Definition 1.12. The tangent space to the $\mathcal{A}_{1}$-orbit of the map-germ is defined to be

$$
T \mathcal{A}_{1} \cdot f=t f\left(\mathbf{m}_{n}^{2} \cdot V\left(\mathbb{R}^{n}\right)\right)+w f\left(\mathbf{m}_{p}^{2} \cdot V\left(\mathbb{R}^{p}\right)\right) .
$$

Unfoldings of map germs. Let $f_{0}:\left(\mathbb{R}^{n}, 0\right) \rightarrow\left(\mathbb{R}^{p}, 0\right)$ be a smooth map-germ.

DeFinition 1.13. A $k$-parameter unfolding of the germ $f_{0}$ is a smooth map-germ $F$ : $\left(\mathbb{R}^{k} \times \mathbb{R}^{n}, 0\right) \rightarrow\left(\mathbb{R}^{k} \times \mathbb{R}^{p}, 0\right)$ such that

1) $\pi_{1} \circ F=\pi_{2}$ where $\pi_{1}: \mathbb{R}^{k} \times \mathbb{R}^{p} \rightarrow \mathbb{R}^{k}, \pi_{2}: \mathbb{R}^{k} \times \mathbb{R}^{n} \rightarrow \mathbb{R}^{k}$ precisely

$$
F(u, x)=(u, f(u, x))
$$

for some smooth $f: \mathbb{R}^{k} \times \mathbb{R}^{n} \rightarrow \mathbb{R}^{p}$,

2) $f_{0}(x)=F(0, x) \quad \forall x \in \mathbb{R}^{n}$.

Definition 1.14. Two $k$-parameter unfoldings $F$ and $G$ of the germ $f_{0} \in \mathbf{m}_{n} . \mathcal{E}_{n, p}$ are said to be isomorphic if there exist germs of diffeomorphisms $\phi \in L_{n, k}$ and $\psi \in L_{p, k}$ such that

$$
G=\psi \circ F \circ \phi^{-1}
$$

where $L_{n, k}$ stands for a $k$-parameter unfolding of identity $i d_{\mathbb{R}^{n}}$,

$$
\begin{gathered}
\phi:\left(\mathbb{R}^{k} \times \mathbb{R}^{n}, 0\right) \rightarrow\left(\mathbb{R}^{k} \times \mathbb{R}^{n}, 0\right), \\
\phi(u, x)=(u, \hat{\phi}(u, x)) \text { where }\left.\hat{\phi}\right|_{\{0\} \times \mathbb{R}^{n}}=i d_{\mathbb{R}^{n}} .
\end{gathered}
$$

DeFinition 1.15. The unfolding is called trivial if it is isomorphic to some constant unfolding $(u, x) \rightarrow\left(u, f_{0}(x)\right)$.

Let $F$ be a $k$-parameter unfolding of $f_{0} \in \mathbf{m}_{n} \cdot \mathcal{E}_{n, p}$ and $h:\left(\mathbb{R}^{q}, 0\right) \rightarrow\left(\mathbb{R}^{k}, 0\right), h(v)=u$ a germ of smooth mapping.

DeFinition 1.16. $q$-parameter unfolding of the germ $f_{0}$ obtained from $F$ in the following way:

$$
G:\left(\mathbb{R}^{q} \times \mathbb{R}^{n}, 0\right) \rightarrow\left(\mathbb{R}^{q} \times \mathbb{R}^{n}, 0\right), \quad G(v, x)=(v, f(h(v), x)),
$$

is called a pullback of $F$ and is denoted by $G=h^{*} F$. 
Definition 1.17. Two unfoldings $F$ and $G$ of the same germ $f_{0}$ are equivalent if $G$ is isomorphic to $h^{*} F$ for some diffeomorphism $h$ of the parameter spaces for $F$ and $G$ respectively.

Definition 1.18. The unfolding $F$ of the germ $f_{0} \in \mathbf{m}_{n} \cdot \mathcal{E}_{n, p}$ is called universal if every other unfolding of $f_{0}$ is isomorphic to $h^{*} F$ for some mapping $h$.

Stability and infinitesimal stability

Definition 1.19. A germ of smooth maping $f:\left(\mathbb{R}^{n}, 0\right) \rightarrow\left(\mathbb{R}^{p}, 0\right)$ is stable if all of its unfoldings are trivial.

DeFinition 1.20. A germ of smooth maping $f$ is infinitesimally stable if

$$
T f=V(f) .
$$

Infinitesimal stability means that for every germ $\xi$ at $0 \in \mathbb{R}^{n}$ of smooth vector field along $f$ there exist germs of vector fields $X \in V\left(\mathbb{R}^{n}\right), Y \in V\left(\mathbb{R}^{p}\right)$ such that

$$
\xi=d f(X)+Y \circ f .
$$

Codimension of the germ

Definition 1.21. If the tangent space to the map-germ $f$ is of finite codimension (over $\mathbb{R}$ ) in $V(f)$ then

$$
\operatorname{codim}_{\mathbb{R}}(f)=\operatorname{dim}_{\mathbb{R}}(V(f) / T f)
$$

is called the codimension of the germ $f$.

If $f$ is $\mathcal{A}$-stable then $\operatorname{codim}_{\mathbb{R}}(f)=0$.

Construction of the universal unfolding. Let $f_{0}:\left(\mathbb{R}^{n}, 0\right) \rightarrow\left(\mathbb{R}^{p}, 0\right)$ be a map-germ of finite codimension $p$. This implies that $V(f) / T f$ is a $p$-dimensional real vector space. Let us assume that equivalence classes $\left[g_{1}\right], \ldots,\left[g_{p}\right]$ span the quotient space $V(f) / T f$. Then

$$
F(u, x)=\left(u, f_{0}(x)+\sum_{i=1}^{p} u_{i} g_{i}(x)\right), \quad u=\left(u_{1}, \ldots, u_{p}\right) \in \mathbb{R}^{p}
$$

is the universal unfolding of the map-germ $f_{0}$.

Determinacy theorems for unipotent subgroups. In the paper [4] the authors have studied actions of unipotent algebraic groups on algebraic varieties and have given a finite determinacy criterion for subgroups whose 1-jets form a unipotent group. Let $\mathbb{F}$ denote the field $\mathbb{R}$ or $\mathbb{C}$ and let $\mathcal{G}$ be one of the groups: $\mathcal{R}, \mathcal{C}, \mathcal{K}, \mathcal{L}, \mathcal{A}$.

Definition 1.22. A subgroup $\mathcal{H}$ of group $\mathcal{G}$ is called strongly closed in $\mathcal{G}$ if $\mathcal{G}_{s} \subset \mathcal{H}$ for some $s$ and $J^{s} \mathcal{H}$ is a closed subgroup in $J^{s} \mathcal{G}$. Moreover if $J^{s} \mathcal{H}$ is an algebraic subgroup then $\mathcal{H}$ is called strongly $Z$-closed.

TheOREm 1.1 ([4], 1.9). Let $\mathcal{G}$ be one of the groups $\mathcal{R}, \mathcal{C}, \mathcal{K}, \mathcal{L}, \mathcal{A}$. Let $\mathcal{H}$ be a strongly $Z$-closed subgroup in $\mathcal{G}$ and $f:\left(\mathbb{F}^{n}, 0\right) \rightarrow\left(\mathbb{F}^{m}, 0\right)$ be a germ of smooth mapping. Then for any finite $r, f$ is $r$-H-determined if and only if there exists a strongly closed subgroup $\mathcal{U} \subset \mathcal{H}$ in $\mathcal{G}$ such that $J^{1} \mathcal{U}$ is unipotent and

$$
\mathbf{m}_{n}^{r+1} \cdot \mathcal{E}_{n, m} \subset L \mathcal{U} . f .
$$


The method of complete transversal. Theorem 1.1 provides a solution for determinacy problems for a very large class of equivalence relations generated by actions of unipotent groups. We may carry out classification inductively on the jet level, i.e. for a given $k$-jet we classify all $(k+1)$-jets. This procedure results in a classification tree whose branches end with sufficient jets. The question remains how to obtain a full list of representatives of classes of $(k+1)$-jets with respect to a given equivalence relation for a given $k$-jet. In the case of unipotent subgroups the answer is given by the complete transversal method [3].

Let $G$ be a Lie group and $X$ a smooth manifold. Let $\phi: G \times X \rightarrow X$ be a smooth action of $G$ on $X$ denoted by $\phi(g, x)=g . x$. By fixing the first argument we obtain the mapping $\phi^{\alpha}: G \rightarrow X$ such that $\phi^{\alpha}(g)=g . \alpha$. The differential of this mapping at the identity of the group $e \in G$ we denote by $\left(d \phi^{\alpha}\right)_{e}: T_{e} G \rightarrow T_{\alpha} X$. With this notation we see that the previously introduced tangent space to the orbit of a $k$-jet of a map-germ $\alpha \in \mathcal{E}_{n, p}$ is the image of $T_{e} G$ by the differential $\left(d \phi^{\alpha}\right)_{e}$, where $G=J^{k} \mathcal{A}$ and $X=J_{n, p}^{k}$.

THEOREM 1.2. Let $G$ be the Lie group acting smoothly on a finite dimensional vector space $V$, and $H$ a vector subspace in $V$ satisfying $g .(\alpha+\beta)=g \cdot \alpha+\beta$ for any $\alpha \in V$ and $\beta \in H$. Then for any $\alpha \in V$ we have $G . \alpha \cap(\{\alpha\}+H) \supseteq\{\alpha\}+\left(T_{\alpha}(G . \alpha) \cap H\right)$. Moreover, if $T$ is a subspace in $H$ such that $T_{\alpha}(G . \alpha)+T \supset H$ then for any $\beta \in H$ the element $\alpha+\beta$ is $G$-equivalent to $\alpha+\beta^{\prime}$ for some $\beta^{\prime} \in T$.

Proof. See [5], 4.10.

The subspace $T$ in the previous theorem is called a complete transversal.

For a given smooth action of a Lie group $G$ on $X$ we define an induced action of elements of Lie algebra on $X$.

Definition 1.23. Let $G$ and $V$ be as in Theorem 1.2 and $\alpha \in V, l \in T_{e} G$. Then we define

$$
l . \alpha=\left(d \phi^{\alpha}\right)_{e}(l), \quad\left(T_{e} G\right) . \alpha=\left\{l . \alpha \mid l \in T_{e} G\right\} .
$$

By the definition of a tangent space to the orbit of a mapping we have:

$$
\left(T_{e} G\right) \cdot \alpha=T_{\alpha}(G \cdot \alpha) \text {. }
$$

THEOREM 1.3. If we replace in Theorem 1.2 the condition $g .(\alpha+\beta)=g . \alpha+\beta$ by the infinitesimal condition $l .(\alpha+\beta)=l . \alpha$ for all $\alpha \in V, \beta \in H, l \in T_{e} G$ then the conclusion of Theorem 1.2 holds.

Proof. See [5], 4.12.

2. The case of $\mathbb{R}^{2} / \mathbb{Z}_{2} \times \mathbb{Z}_{2}$. In the previous section we have discussed the problem of classification of projections of surfaces without boundary as well as with a smooth boundary. The aim of this section is to consider the case of a surface with singular boundary. As the structure of possible singularities of the boundary may be extremely complicated we restrict further analysis to the simplest possible case where the boundary consists of two transversally intersecting lines. The local model of such a surface is the quotient space $\mathbb{R}^{2} / \mathbb{Z}_{2} \times \mathbb{Z}_{2}$.

Statement of the problem. Let $X$ be a germ at zero of a surface with singular boundary. Therefore $X$ is locally diffeomorphic to the quotient space $\mathbb{R}^{2} / \mathbb{Z}_{2} \times \mathbb{Z}_{2}$. As a consequence 
we can identify $X$ with the germ of $\left\{(x, y) \in \mathbb{R}^{2}: x \geq 0, y \geq 0\right\}$. Our goal is to classify map-germs $f:(X, 0) \rightarrow\left(\mathbb{R}^{2}, 0\right)$ up to diffeomorphisms preserving the singular boundary of the surface as well as its interior and arbitrary diffeomorphisms preserving zero in the target.

Description of the group $\mathcal{B}_{R}$ acting in the source. Let $\mathcal{B}_{R} \subset \mathcal{D}(2)$ be a subgroup of germs of diffeomorphisms of the source. Let $(x, y)$ be the local coordinate system at $0 \in \mathbb{R}^{2}$. Then $f \in \mathcal{B}_{R}$ may be written as $f(x, y)=\left(f_{1}(x, y), f_{2}(x, y)\right)$ for some $f_{1}, f_{2} \in \mathcal{E}_{2}$. The boundary preserving conditions give the following relations: $f(x, 0)=\left(f_{1}(x, y), 0\right)$ and $f(0, y)=\left(0, f_{2}(x, y)\right)$. It follows that there exist germs of functions $\hat{f}_{1}, \hat{f}_{2} \in \mathcal{E}_{2}$ such that: $f_{1}(x, y)=x \hat{f}_{1}(x, y)$ and $f_{2}(x, y)=y \hat{f}_{2}(x, y)$. The interior preserving condition reads: $f_{1}(x, y)>0$ for $x>0 \Rightarrow \hat{f}_{1}(x, y)>0$ and $f_{2}(x, y)>0$ for $y>0 \Rightarrow \hat{f}_{2}(x, y)>0$. Because $f$ is a local diffeomorphism at $0 \in \mathbb{R}^{2}$ we have

$$
\operatorname{det}(d f(0,0))=\hat{f}_{1}(0,0) \hat{f}_{2}(0,0) \neq 0 .
$$

The subgroup $\mathcal{B}_{R}$ consists of diffeomorphisms of the form $(x, y) \rightarrow\left(x \hat{f}_{1}(x, y), y \hat{f}_{2}(x, y)\right)$, $\hat{f}_{1}(x, y)>0$ for $x>0, \hat{f}_{2}(x, y)>0$ for $y>0, \hat{f}_{1}(0,0) \hat{f}_{2}(0,0) \neq 0$.

Algebraic structure of $\mathcal{B}$. Let $\mathcal{B}_{L}=\mathcal{D}(2)$. Then the product group $\mathcal{B}=\mathcal{B}_{L} \times \mathcal{B}_{R}$ acts on the space of map-germs $f:(X, 0) \rightarrow\left(\mathbb{R}^{2}, 0\right)$ resulting in the required equivalence relation. In terms of group actions our task is to classify the orbits of the action of $\mathcal{B}$ of low codimension.

Tangent space to the $\mathcal{B}_{1}$-orbit of a map-germ

Proposition 2.1. Let $\mathcal{B}_{1}$ be the subgroup in $\mathcal{B}$ consisting of those map-germs whose 1 jets are identity. Then the tangent space to the $\mathcal{B}_{1}$-orbit of a map-germ has the following structure:

$$
L \mathcal{B}_{1} \cdot f=\mathbf{m}_{2}\left\{x \frac{\partial f}{\partial x}, y \frac{\partial f}{\partial y}\right\}+\left(f^{*} \mathbf{m}_{2}\right)^{2}\left\{e_{1}, e_{2}\right\} .
$$

Using generators of the maximal ideal $\mathbf{m}_{2}$ and a pullback $f^{*} \mathbf{m}_{2}$ the last can be written as

$$
L \mathcal{B}_{1} . f=\mathcal{E}_{2}\left\{x^{2} \frac{\partial f}{\partial x}, x y \frac{\partial f}{\partial x}, x y \frac{\partial f}{\partial y}, y^{2} \frac{\partial f}{\partial y}\right\}+f^{*} \mathcal{E}_{2}\left\{f_{1}^{2}, f_{1} f_{2}, f_{2}^{2}\right\}\left\{e_{1}, e_{2}\right\} .
$$

Theorems on k-determinacy

THEOREM 2.1. Let $\mathcal{B}$ be the considered subgroup of $\mathcal{A}$. Let $\mathcal{B}_{1} \subset \mathcal{U} \subset \mathcal{B}$ be subgroups in $\mathcal{B}$ such that 1 -jets of map-germs from $\mathcal{B}_{1}$ are identities and $J^{1} \mathcal{U}$ is a unipotent group. If for some map-germ $f:(X, 0) \rightarrow\left(\mathbb{R}^{2}, 0\right)$

$$
\mathbf{m}_{2}^{r+1} \cdot \mathcal{E}_{2,2} \subset L U . f
$$

then $f$ is $r$ - $\mathcal{B}$-determined.

Proposition 2.2. If $f:(X, 0) \rightarrow\left(\mathbb{R}^{2}, 0\right)$ satisfies

$$
\begin{aligned}
& \mathbf{m}_{2}^{l} \cdot \mathcal{E}_{2,2} \subset \mathcal{E}_{2}\left\{x \frac{\partial f}{\partial x}, y \frac{\partial f}{\partial y}\right\}+f^{*} \mathbf{m}_{2} \cdot \mathcal{E}_{2,2}+\mathbf{m}_{2}^{l+1} \cdot \mathcal{E}_{2,2}, \\
& \mathbf{m}_{2}^{r+1} \cdot \mathcal{E}_{2,2} \subset L \mathcal{B}_{1} \cdot f+\mathbf{m}_{2}^{r+l+1} \cdot \mathcal{E}_{2,2}
\end{aligned}
$$

then $f$ is $r$ - $\mathcal{B}_{1}$-determined. 
The last proposition is an algebraic criterion for checking if a given $r$-jet is in fact sufficient. The proof requires some algebraic lemma about finitely generated modules.

Lemma 2.1 ([4], 2.6). Let $C$ be a finitely generated $\mathcal{E}_{n}$-module, $B \subset C$ a finitely generated $\mathcal{E}_{n}$-submodule, $A \subset f^{*} \mathbf{m}_{p}$.C a finitely generated $\mathcal{E}_{p}$-module (via $f$ ) and $M$ a proper, finitely generated ideal in $\mathcal{E}_{n}$. If

$$
M . C \subset A+B+M \cdot\left(f^{*} \mathbf{m}_{p}+M\right) \cdot C
$$

then

$$
\text { M.C } \subset A+B .
$$

Proof of Proposition 2.2. We know that

$$
L \mathcal{B}_{1} \cdot f=\mathbf{m}_{2}\left\{x \frac{\partial f}{\partial x}, y \frac{\partial f}{\partial y}\right\}+\left(f^{*} \mathbf{m}_{2}\right)^{2}\left\{e_{1}, e_{2}\right\} .
$$

Substituting formula (2.5) into the inclusion (2.4) gives

$$
\begin{aligned}
\mathbf{m}_{2}^{r+1} \cdot \mathcal{E}_{2,2} \subset & \mathbf{m}_{2}\left\{x \frac{\partial f}{\partial x}, y \frac{\partial f}{\partial y}\right\}+\left(f^{*} \mathbf{m}_{2}\right)^{2}\left\{e_{1}, e_{2}\right\} \\
& +\mathbf{m}_{2}^{r+1}\left(\mathcal{E}_{2}\left\{x \frac{\partial f}{\partial x}, y \frac{\partial f}{\partial y}\right\}+f^{*} \mathbf{m}_{2} \cdot \mathcal{E}_{2,2}\right) \\
= & \mathbf{m}_{2}\left\{x \frac{\partial f}{\partial x}, y \frac{\partial f}{\partial y}\right\}+\mathbf{m}_{2}^{r+1}\left\{x \frac{\partial f}{\partial x}, y \frac{\partial f}{\partial y}\right\}+\left(f^{*} \mathbf{m}_{2}\right)^{2}\left\{e_{1}, e_{2}\right\} \\
& +\mathbf{m}_{2}^{r+1} \cdot f^{*} \mathbf{m}_{2} \cdot \mathcal{E}_{2,2} \\
= & \mathbf{m}_{2}\left\{x \frac{\partial f}{\partial x}, y \frac{\partial f}{\partial y}\right\}+\left(f^{*} \mathbf{m}_{2}\right)^{2}\left\{e_{1}, e_{2}\right\}+\mathbf{m}_{2}^{r+1} \cdot f^{*} \mathbf{m}_{2} \cdot \mathcal{E}_{2,2} \\
\subset & L \mathcal{B}_{1} \cdot f+\mathbf{m}_{2}^{r+1} \cdot\left(f^{*} \mathbf{m}_{2} \cdot \mathcal{E}_{2,2}+\mathbf{m}_{2}^{r+1} \cdot \mathcal{E}_{2,2}\right) \\
= & L \mathcal{B}_{1} \cdot f+\mathbf{m}_{2}^{r+1} \cdot\left(f^{*} \mathbf{m}_{2} \cdot \mathcal{E}_{2}+\mathbf{m}_{2}^{r+1}\right) \cdot \mathcal{E}_{2,2} .
\end{aligned}
$$

Next we apply lemma 2.1 with $A=\left(f^{*} \mathbf{m}_{2}\right)^{2}\left\{e_{1}, e_{2}\right\}, B=\mathbf{m}_{2}\left\{x \frac{\partial f}{\partial x}, y \frac{\partial f}{\partial y}\right\}, M=\mathbf{m}_{2}^{r+1}$, $C=\mathcal{E}_{2,2}, C_{n}=\mathcal{E}_{2}$ and we obtain that $\mathbf{m}_{2}^{r+1} \mathcal{E}_{2,2} \subset L \mathcal{B}_{1} . f$. Hence $f$ is finitely $r-\mathcal{B}_{1}$ determined.

Theorems on complete transversals

THEOREM 2.2. Let $g$ be a $k$-jet in $J_{2,2}^{2}$ and $T$ a vector subspace in $H_{2,2}^{k+1}$, the space of homogeneous mappings $\mathbb{R}^{2} \rightarrow \mathbb{R}^{2}$ of degree $k+1$ and

$$
L\left(J^{k+1} \mathcal{B}_{1}\right) \cdot g+T \supset H_{2,2}^{k+1} .
$$

Then any $(k+1)$-jet $f$ whose $k$-jet is equal to $g$ is $\mathcal{B}_{1}$-equivalent to $g+t$ for some $t \in T$.

The subspace $T$ is called a complete transversal.

Proof. Notice that this theorem is a special case of Theorem 1.2 where the Lie group is the group of $(k+1)$-jets of diffeomorphisms whose 1-jets are identity. This group is denoted by $J^{k}\left(\mathcal{B}_{1}\right)$. The vector space is the space of all $(k+1)$-jets denoted by $J_{2,2}^{k}$ and a vector subspace is $H^{k+1}(2,2)$. By Theorem 1.3 it's enough to show the infinitesimal 
condition

$$
\text { l. }(\alpha+\beta)=l . \alpha \quad \forall \alpha \in J_{2,2}^{k}, \beta \in H^{k+1}(2,2), l \in T_{e}\left(J^{k+1} \mathcal{B}_{1}\right) .
$$

In our notation this condition has the form

$$
l .(f+F)=l . f \quad \forall f \in J_{2,2}^{k}, F \in H^{k+1}(2,2), l \in L\left(J^{k+1} \mathcal{B}_{1}\right) .
$$

Recall that

$$
L\left(J^{k+1} \mathcal{B}_{1}\right) \cdot f=j^{k+1}\left(\mathbf{m}_{2}\left\{x \frac{\partial f}{\partial x}, y \frac{\partial f}{\partial y}\right\}\right)+j^{k+1}\left(f^{*} \mathbf{m}_{2}^{2}\left\{e_{1}, e_{2}\right\}\right) .
$$

Since only the right component (which has to preserve two intersecting lines in the source) of the $\mathcal{B}$ group is different from $\mathcal{A}$ we show the condition (2.7) only for the part of $\mathcal{B}$ acting in the source. Let us consider any element $l \in \mathcal{R} \mathcal{B}_{1}$, the Lie algebra of right equivalence group. Its action on $(f+F)$ may be written for some $h_{1}, h_{2} \in \mathbf{m}_{2}$ in the following way

$$
\begin{aligned}
l .(f+F) & =j^{k+1}\left(h_{1} x \frac{\partial(f+F)}{\partial x}+h_{2} y \frac{\partial(f+F)}{\partial y}\right) \\
& =j^{k+1}\left(h_{1} x \frac{\partial f}{\partial x}+h_{1} x \frac{\partial F}{\partial x}+h_{2} y \frac{\partial f}{\partial y}+h_{2} y \frac{\partial F}{\partial y}\right) \\
& =j^{k+1}\left(h_{1} x \frac{\partial f}{\partial x}+h_{2} y \frac{\partial f}{\partial y}\right) \\
& =l . f .
\end{aligned}
$$

The last equality holds because $h_{1} x \frac{\partial F}{\partial x}, h_{2} y \frac{\partial F}{\partial y} \in \mathbf{m}_{2}^{k+2}$.

3. Classification calculations. In this section we carry out classification inductively on jet level. We restrict our discussion to map-germs of corank $\leq 1$ and codim $\leq 3$. From now on the symbol $\sim$ means $J^{k} \mathcal{B}$-equivalence. Sufficient jets will be marked by ${ }^{(*)}$. Each of symbols $\varepsilon_{1}, \varepsilon_{2}, \ldots$ will denote independently \pm 1 . We begin with classification of 1 -jets.

3.1. 1-jets. Suppose $j^{1}(f)=\left(a_{1} x+a_{2} y, b_{1} x+b_{2} y\right)$. We distinguish the following possibilities:

a) If $\operatorname{det}\left(\begin{array}{ll}a_{1} & a_{2} \\ b_{1} & b_{2}\end{array}\right) \neq 0$ then $j^{1}(f) \sim(x, y)$

b) If $\operatorname{det}\left(\begin{array}{ll}a_{1} & a_{2} \\ b_{1} & b_{2}\end{array}\right)=0$ and $\left(a_{1} \neq 0\right.$ or $\left.b_{1} \neq 0\right)$ then $\left(\begin{array}{cc}a_{1} & a_{2} \\ b_{1} & b_{2}\end{array}\right) \sim\left(\begin{array}{cc}1 & \gamma \\ 0 & 0\end{array}\right)$ where $\gamma=\frac{a_{2}}{a_{1}}$ if $a_{1} \neq 0$ or $\gamma=\frac{b_{2}}{b_{1}}$ if $b_{1} \neq 0$. Therefore $j^{1}(f) \sim\left(x+\frac{a_{2}}{a_{1}} y, 0\right)$ or $j^{1}(f) \sim\left(x+\frac{b_{2}}{b_{1}} y, 0\right)$. Next, depending on the sign of $\gamma$ we have the following 3 cases: $j^{1}(f) \sim(x, 0)$ or $j^{1}(f) \sim(x \pm y, 0)$.

c) If $\operatorname{det}\left(\begin{array}{ll}a_{1} & a_{2} \\ b_{1} & b_{2}\end{array}\right)=0 \wedge\left(a_{2} \neq 0 \vee b_{2} \neq 0\right)$ then by symmetry we have $j^{1}(f) \sim$ $(x \pm y, 0)$ or $j^{1}(f) \sim(y, 0)$.

d) The last case is $j^{1}(f)=(0,0)$. As $j^{1}(f)$ is of corank 2 it cannot arise as a projection of 2-surface onto 2 -space.

Finally every 1 -jet is $J^{1} \mathcal{B}$-equivalent to one of the following five forms:

$j^{1}(f)=(x, y)($ see 3.1 .1$)$, 
$j^{1}(f)=(x, 0)($ see 3.1 .2$)$,

$j^{1}(f)=(y, 0)($ see 3.1 .3$)$,

$j^{1}(f)=(x \pm y, 0)($ see 3.1.4).

3.1.1. Case $j^{1}(f)=(x, y)^{(*)}$. This form is $\mathcal{B}$-stable.

3.1.2. Case $j^{1}(f)=(x, 0)$. The complete transversal is $T=\left\{\left(y^{2}, 0\right),(0, x y),\left(0, y^{2}\right)\right\}$ so every 2 -jet with $j^{1}(f)=(x, 0)$ is $J^{2} \mathcal{B}$-equivalent to $j^{2}(f)=\left(x+a_{1} y^{2}, a_{2} x y+a_{3} y^{2}\right)$ for some $a_{1}, a_{2}, a_{3} \in \mathbb{R}^{3}$. We have various cases to consider. We mainly use simple linear coordinate changes in further simplifications:

A) For $a_{3} \neq 0, j^{2}(f)=\left(x+a_{1} y^{2}, a_{2} x y+a_{3} y^{2}\right) \sim\left(x-\frac{a_{1} a_{2}}{a_{3}} x y, a_{2} x y+a_{3} y^{2}\right)$.

A1) For $a_{2} \neq 0, j^{2}(f) \sim\left(x, x y \pm y^{2}\right)($ see 3.2.1).

A2) For $a_{2}=0, j^{2}(f) \sim\left(x, y^{2}\right)($ see 3.2.2).

B) For $a_{3}=0, j^{2}(f)=\left(x+a_{1} y^{2}, a_{2} x y\right)$.

B1) For $a_{1} \neq 0$ and $a_{2} \neq 0 . j^{2}(f) \sim\left(x \pm y^{2}, x y\right)$ (see 3.2.3).

B2) For $a_{1} \neq 0$ and $a_{2}=0, j^{2}(f) \sim\left(x \pm y^{2}, 0\right), \operatorname{codim}_{\mathbb{R}}\left(j^{2}(f)\right)=4$.

B3) For $a_{1}=0$ and $a_{2} \neq 0, j^{2}(f) \sim(x, x y), \operatorname{codim}_{\mathbb{R}}\left(j^{2}(f)\right)=4$.

B4) For $a_{1}=0$ and $a_{2}=0, j^{2}(f)=(x, 0), \operatorname{codim}_{\mathbb{R}}\left(j^{2}(f)\right)=5$.

3.1.3. Case $j^{1}(f)=(y, 0)$.

$$
\begin{gathered}
L\left(J^{2} \mathcal{B}_{1}\right) \cdot f=J^{2}\left(\mathcal{E}_{2}\left\langle\left(\begin{array}{c}
y^{2} \\
0
\end{array}\right),\left(\begin{array}{c}
x y \\
0
\end{array}\right)\right\rangle\right)+J^{2}\left(f^{*} \mathcal{E}_{2}\left\langle y^{2}\right\rangle\left\{e_{1}, e_{2}\right\}\right), \\
L\left(J^{2} \mathcal{B}_{1}\right) \cdot f=\left\langle\left(\begin{array}{c}
y^{2} \\
0
\end{array}\right),\left(\begin{array}{c}
x y \\
0
\end{array}\right)\right\rangle_{\mathbb{R}}+\left\langle y^{2}\right\rangle_{\mathbb{R}}\left\{e_{1}, e_{2}\right\} .
\end{gathered}
$$

The complete transversal condition: $L\left(J^{2} \mathcal{B}_{1}\right) \cdot f+T \supset H^{2}(2,2)$ implies that the only missing homogeneous terms of order 2 are: $\left(x^{2}, 0\right),(0, x y),\left(0, x^{2}\right)$.

The transversal is $T=\left\{\left(x^{2}, 0\right),(0, x y),\left(0, x^{2}\right)\right\}$ and every 2-jet with 1 -jet $(y, 0)$ is $J^{2} \mathcal{B}$-equivalent to

$$
j^{2}(f)=\left(y+a_{1} x^{2}, a_{2} x y+a_{3} x^{2}\right) .
$$

One can easily see that this case and $j^{1}(f)=(x, 0)$ have isomorphic tangent spaces and complete transversals as well. The isomorphism is given by the symmetric change of variables $(x \rightarrow y, y \rightarrow x)$. This fact will be used at all levels of classification so at the end of the classification we have to add to the resulting list of normal forms those which arise by the symmetric change of variables from germs whose 1-jet equals $(x, 0)$.

Proposition 3.1. If $f \in \mathcal{E}_{2,2}$ then the $\mathcal{B}_{1}$-tangent spaces to $f(x, y)$ and $f(y, x)$ are isomorphic.

Proof. Let $g \in \mathcal{E}_{2,2}$ be such that $g(x, y)=f(y, x)$ and $T: \mathbb{R}^{2} \rightarrow \mathbb{R}^{2}, T(x, y)=(y, x)$ be the reflection mapping. Then $g$ may be written in the form $g(x, y)=(f \circ T)(x, y)$. We prove the following: $L \mathcal{B}_{1} . f \stackrel{i s o}{\cong} L \mathcal{B}_{1} . g$. Let $X, Y \in \mathcal{E}_{2}, X(x, y)=x, Y(x, y)=y$ be the coordinate functions. As $T$ induces a homomorphism of rings $T^{*}: \mathcal{E}_{2} \rightarrow \mathcal{E}_{2}$, then $T^{*} X=Y, T^{*} Y=X$. The differential $d T$ is nonsingular at the origin, so $T$ is a local 
diffeomorphism on some neighbourhood of $(0,0)$ and $T^{*}$ is an automorphism of the ring $\mathcal{E}_{2}$. By the identification $\mathcal{E}_{2,2}=\mathcal{E}_{2} \oplus \mathcal{E}_{2}$ we get a canonical lift of $T^{*}$ to an automorphism of the module $\mathcal{E}_{2,2}$. Consequently, we may regard $T^{*}\left(L \mathcal{B}_{1} . f\right)$ as the automorphic image of the tangent space $L \mathcal{B}_{1} . f$.

3.1.4. Case $j^{1}(f)=\left(x+\varepsilon_{1} y, 0\right)$.

$$
\begin{gathered}
L\left(J^{2} \mathcal{B}_{1}\right) . f=J^{2}\left(\mathcal{E}_{2}\left\langle\left(\begin{array}{c}
x^{2} \\
0
\end{array}\right),\left(\begin{array}{c}
x y \\
0
\end{array}\right),\left(\begin{array}{c}
\epsilon x y \\
0
\end{array}\right),\left(\begin{array}{c}
\epsilon y^{2} \\
0
\end{array}\right)\right\rangle\right)+J^{2}\left(f^{*} \mathcal{E}_{2}\langle x+\epsilon y\rangle\left\{e_{1}, e_{2}\right\}\right), \\
L\left(J^{2} \mathcal{B}_{1}\right) . f=\left\langle\left(\begin{array}{c}
x^{2} \\
0
\end{array}\right),\left(\begin{array}{c}
x y \\
0
\end{array}\right),\left(\begin{array}{c}
\epsilon x y \\
0
\end{array}\right),\left(\begin{array}{c}
\epsilon y^{2} \\
0
\end{array}\right)\right\rangle_{\mathbb{R}}+\left\langle x^{2}+2 \epsilon x y+y^{2}\right\rangle_{\mathbb{R}}\left\{e_{1}, e_{2}\right\} .
\end{gathered}
$$

The complete transversal is $T=\left\{\left(0, x^{2}\right),(0, x y)\right\}$ so every 2 -jet with 1 -jet $(x, 0)$ is $J^{2} \mathcal{B}$ equivalent to

$$
j^{2}(f)=\left(x+\varepsilon_{1} y, a_{1} x y+a_{2} x^{2}\right) .
$$

We have the following subcases:

A) If $a_{1} \neq 0$ then $j^{2}(f) \sim\left(x+\epsilon y, x y+\frac{a_{2}}{a_{1}} x^{2}\right)$.

A1) If $a_{2} \neq 0$ then $j^{2}(f) \sim\left(x+\varepsilon_{1} y, x y+\gamma x^{2}\right)$ (see 3.2.4), where $\gamma=\frac{a_{2}}{a_{1}}$ is a modulus.

A2) If $a_{2}=0$ then $j^{2}(f) \sim\left(x+\varepsilon_{1} y, x y\right)$ (see 3.2.5).

B) If $a_{1}=0$ then $\left(x+\varepsilon_{1} y, a_{1} x y+a_{2} x^{2}\right)=\left(x+\varepsilon_{1} y, a_{2} x^{2}\right)$.

B1) If $a_{2} \neq 0$ then $j^{2}(f) \sim\left(x+\varepsilon_{1} y, x^{2}\right)$ (see 3.2.6).

B2) If $a_{2}=0$ then $j^{2}(f) \sim\left(x+\varepsilon_{1} y, 0\right)$ (see 3.2.7).

3.2. 2-Jets

3.2.1. Case $j^{2}(f)=\left(x, x y+\varepsilon_{2} y^{2}\right)$. We calculate the tangent space in 3 -jets.

$$
\begin{aligned}
L\left(J^{3} \mathcal{B}_{1}\right) \cdot f= & \left\langle\left(\begin{array}{c}
x^{2} \\
x^{2} y
\end{array}\right),\left(\begin{array}{c}
x^{3} \\
0
\end{array}\right),\left(\begin{array}{c}
x^{2} y \\
0
\end{array}\right),\left(\begin{array}{c}
x y^{2} \\
0
\end{array}\right),\left(\begin{array}{c}
x y \\
x y^{2}
\end{array}\right),\left(\begin{array}{c}
0 \\
x^{2} y+2 \varepsilon_{2} x y^{2}
\end{array}\right),\left(\begin{array}{c}
0 \\
x y^{2}+\varepsilon_{y}^{3}
\end{array}\right)\right\rangle_{\mathbb{R}} \\
& +\left\langle x^{2}, x^{3}, x^{2} y+\varepsilon_{2} x y^{2}\right\rangle_{\mathbb{R}}\left\{e_{1}, e_{2}\right\} .
\end{aligned}
$$

By the complete transversal condition $L\left(J^{3} \mathcal{B}_{1}\right) \cdot f+T \supset H^{3}(2,2)$ we seek a minimal vector subspace $T$ such that the tangent space $L\left(J^{3} \mathcal{B}_{1}\right) . f$ together with $T$ contains all the homogeneous mappings of degree 3. Because the elements

$$
\left(\begin{array}{c}
x^{3} \\
0
\end{array}\right),\left(\begin{array}{c}
x^{2} y \\
0
\end{array}\right),\left(\begin{array}{c}
x y^{2} \\
0
\end{array}\right),\left(\begin{array}{c}
0 \\
x^{3}
\end{array}\right)
$$

belong to $L\left(J^{3} \mathcal{B}_{1}\right) . f$, for the remaining cases we take

$$
\left(\begin{array}{c}
0 \\
x^{2} y+2 \varepsilon_{2} x y^{2}
\end{array}\right),\left(\begin{array}{c}
0 \\
x^{2} y+\varepsilon_{2} x y^{2}
\end{array}\right) .
$$

We see that $\operatorname{det}\left(\begin{array}{cc}1 & 2 \\ 1 & \varepsilon_{2}\end{array}\right) \neq 0$ so $\left\{\left(\begin{array}{c}0 \\ x^{2} y\end{array}\right),\left(\begin{array}{c}0 \\ x y^{2}\end{array}\right)\right\}$ also belong to the tangent space. The mapping $\left(\begin{array}{c}0 \\ y^{3}\end{array}\right)$ comes from the right tangent space:

$$
\left(\begin{array}{c}
0 \\
x y^{2}+2 \varepsilon_{2} y^{3}
\end{array}\right)-\left(\begin{array}{c}
0 \\
x y^{2}
\end{array}\right)=\left(\begin{array}{c}
0 \\
2 \varepsilon_{2} y^{3}
\end{array}\right)
$$


The only homogeneous term of degree 3 we cannot obtain is $\left(\begin{array}{c}y^{3} \\ 0\end{array}\right)$ so a complete transversal is $T=\left\{\left(y^{3}, 0\right)\right\}$ and every 3 -jet with 2 -jet $\left(x, x y+\varepsilon_{2} y^{2}\right)$ is $J^{3} \mathcal{B}$-equivalent to

$$
j^{3}(f)=\left(x+a_{1} y^{3}, x y+\varepsilon_{2} y^{2}\right) .
$$

Now by simple reduction we get the following cases:

A) For $a_{1} \neq 0$ we get $j^{3} f \sim\left(x+\varepsilon_{1} y^{3}, x y+\varepsilon_{2} y^{2}\right)$ (see 3.3.1).

B) For $a_{1}=0$ we get $j^{3} f \sim\left(x, x y+\varepsilon_{2} y^{2}\right)$ (see 3.3.2).

3.2.2. Case $j^{2}(f)=\left(x, y^{2}\right)$.

$$
\begin{gathered}
L\left(J^{3} \mathcal{B}_{1}\right) . f=J^{3}\left(\mathcal{E}_{2}\left\langle\left(\begin{array}{c}
x^{2} \\
0
\end{array}\right),\left(\begin{array}{c}
x y \\
0
\end{array}\right),\left(\begin{array}{c}
0 \\
2 x y^{2}
\end{array}\right),\left(\begin{array}{c}
0 \\
2 y^{3}
\end{array}\right)\right\rangle\right)+J^{3}\left(f^{*} \mathcal{E}_{2}\left\langle x^{2}, x y^{2}, y^{4}\right\rangle\left\{e_{1}, e_{2}\right\}\right), \\
L\left(J^{3} \mathcal{B}_{1}\right) \cdot f=\left\langle\left(\begin{array}{c}
x^{2} \\
0
\end{array}\right),\left(\begin{array}{c}
x y \\
0
\end{array}\right),\left(\begin{array}{c}
0 \\
2 x y^{2}
\end{array}\right),\left(\begin{array}{c}
0 \\
2 y^{3}
\end{array}\right)\right\rangle_{\mathbb{R}}+\left\langle x^{2}, x^{3}, x y^{2}\right\rangle_{\mathbb{R}}\left\{e_{1}, e_{2}\right\} .
\end{gathered}
$$

From the complete transversal condition $L\left(J^{3} \mathcal{B}_{1}\right) \cdot f+T \supset H^{3}(2,2)$ it follows that $T=$ $\left\{\left(y^{3}, 0\right),\left(0, x^{2} y\right)\right\}$ so every 3 -jet with 2 -jet $\left(x, y^{2}\right)$ is $J^{3} \mathcal{B}$-equivalent to

$$
j^{3}(f)=\left(x+a_{1} y^{3}, y^{2}+a_{2} x^{2} y\right) .
$$

A1) For $a_{1} \neq 0$ and $a_{2} \neq 0$ by scaling transformation we get $j^{3}(f) \sim\left(x+\varepsilon_{1} y^{3}, y^{2}+\varepsilon_{2} x^{2} y\right)$ This case is of codimension 4 however it is sufficient. One may prove 3-determinacy using criterion 2.2 with $l=2$ and $r=3$.

A2) For $a_{1} \neq 0$ and $a_{2}=0, j^{3}(f) \sim\left(x+\varepsilon_{1} y^{3}, y^{2}\right), \operatorname{codim}_{\mathbb{R}}\left(j^{3}(f)\right)=4$.

A3) For $a_{1}=0$ and $a_{2} \neq 0, j^{3}(f) \sim\left(x, y^{2}+\varepsilon_{2} x^{2} y\right), \operatorname{codim}_{\mathbb{R}}\left(j^{3}(f)\right)=4$.

A4) For $a_{1}=0$ and $a_{2}=0, j^{3}(f) \sim\left(x, y^{2}\right), \operatorname{codim}_{\mathbb{R}}\left(j^{3}(f)\right)=5$.

3.2.3. Case $j^{2}(f)=\left(x+\varepsilon_{1} y^{2}, x y\right)$.

$$
\begin{aligned}
L\left(J^{3} \mathcal{B}_{1}\right) . f= & \left\langle\left(\begin{array}{c}
x^{2} \\
x^{2} y
\end{array}\right),\left(\begin{array}{c}
x^{3} \\
0
\end{array}\right),\left(\begin{array}{c}
x^{2} y \\
0
\end{array}\right),\left(\begin{array}{c}
x y^{2} \\
0
\end{array}\right),\left(\begin{array}{c}
x y \\
x y^{2}
\end{array}\right),\left(\begin{array}{c}
2 \varepsilon_{1} x y^{2} \\
x^{2} y
\end{array}\right),\left(\begin{array}{c}
2 \varepsilon_{1} y^{3} \\
x y^{2}
\end{array}\right)\right\rangle_{\mathbb{R}} \\
& +\left\langle x^{2}+2 \varepsilon_{1} x y^{2}, x^{3}, x^{2} y\right\rangle_{\mathbb{R}}\left\{e_{1}, e_{2}\right\}
\end{aligned}
$$

From the complete transversal condition $L\left(J^{3} \mathcal{B}_{1}\right) \cdot f+T \supset H^{3}(2,2)$ it follows that $T=$ $\left\{\left(y^{3}, 0\right),\left(0, y^{3}\right)\right\}$ so every 3 -jet with 2 -jet $\left(x+\varepsilon_{1} y^{2}, x y\right)$ is $J^{3} \mathcal{B}$-equivalent to

$$
j^{3}(f)=\left(x+\varepsilon_{1} y^{2}+a_{1} y^{3}, x y+a_{2} y^{3}\right) .
$$

A1) For $a_{1} \neq 0$ and $a_{2} \neq 0$ we apply the sequence of transformations: $\left(x \rightarrow\left|a_{2}\right| \beta^{2} x\right.$, $y \rightarrow \beta y)$ for $\beta=\left|a_{1}\right|^{-1},\left(u \rightarrow a_{1}^{2} u, v \rightarrow\left|a_{1}\right|^{3} /\left|a_{2}\right| v\right),\left(u \rightarrow u-\varepsilon_{2} \varepsilon_{3} v, v \rightarrow v\right)$, $\left(x \rightarrow x /\left(\left|a_{2}\right|-\varepsilon_{2} \varepsilon_{3} y\right)\right),\left(v \rightarrow\left|a_{2}\right| v\right),\left(x \rightarrow a_{2}^{2} x, y \rightarrow\left|a_{2}\right| y\right)$. We finally arrive at the form

$$
j^{3}(f) \sim\left(x+\varepsilon_{1} y^{2}, x y+\varepsilon_{1} \varepsilon_{2} x y^{2}+a_{2} y^{3}\right)
$$

with $\operatorname{codim}_{\mathbb{R}}(f)=4$ for all values of the modulus $a_{2} \neq 0$.

A2) For $a_{1} \neq 0$ and $a_{2}=0$ we apply set transformations: $\left(x \rightarrow x, y \rightarrow y\left(1-\frac{a_{1}}{2 \varepsilon_{1}} y\right)\right)$, $\left(x \rightarrow \beta^{2} x, y \rightarrow \beta y\right), \beta=2 /\left|a_{1}\right|\left(u \rightarrow u\left|a_{1}\right|^{2} / 4, v \rightarrow v\left|a_{1}\right|^{3} / 8\right)$. We get the form

$$
j^{3}(f) \sim\left(x+\varepsilon_{1} y^{2}, x y-\varepsilon_{1} \varepsilon_{2} x y^{2}\right)
$$

with $\operatorname{codim}_{\mathbb{R}}(f)=4$. 
A3) For $a_{1}=0$ and $a_{2} \neq 0, j^{3}(f)=\left(x+\varepsilon_{1} y^{2}, x y+a_{2} y^{3}\right), \operatorname{codim}_{\mathbb{R}}(f)=4$, for all values of the modulus $a_{2} \neq 0$.

A4) For $a_{1}=0$ and $a_{2}=0, j^{3}(f)=\left(x+\varepsilon_{1} y^{2}, x y\right), \operatorname{codim}_{\mathbb{R}}(f)=4$,

3.2.4. Case $j^{2}(f)=\left(x+\varepsilon_{1} y, x y+\gamma x^{2}\right)^{(*)}$. Codimension analysis in 2-jet space shows that for $\gamma \neq 0, \operatorname{codim}_{\mathbb{R}}\left(j^{2}(f)\right)=2$. The complete transversal in 3-jets is empty for $\gamma \neq 0, \frac{1}{2} \varepsilon_{1}, \varepsilon_{1}$. One may expect that $j^{2} f$ is 2 - $\mathcal{B}$-sufficient. We use determinacy conditions 2.2 with $l=2, r=2$. The determinacy conditions which have to be satisfied are:

$$
\begin{aligned}
& \mathbf{m}_{2}^{2} \cdot \mathcal{E}_{2,2} \subset \mathcal{E}_{2}\left\{x \frac{\partial f}{\partial x}, y \frac{\partial f}{\partial y}\right\}+f^{*} \mathbf{m}_{2} \cdot \mathcal{E}_{2,2}+\mathbf{m}_{2}^{3} \cdot \mathcal{E}_{2,2}, \\
& \mathbf{m}_{2}^{3} \cdot \mathcal{E}_{2,2} \subset L \mathcal{B}_{1} \cdot f+\mathbf{m}_{2}^{5} \cdot \mathcal{E}_{2,2} .
\end{aligned}
$$

The first condition is fulfilled provided that $\gamma \neq 0, \frac{1}{2} \varepsilon_{1}, \varepsilon_{1}$. This is because

$$
\begin{gathered}
\left(x+\varepsilon_{1} y\right)^{2}=f_{1}^{2} \in f^{*} \mathbf{m}_{2}, \\
\left(x+\varepsilon_{1} y\right)=f_{1} \in f^{*} \mathbf{m}_{2} \Rightarrow\left(x+\varepsilon_{1} y\right)\left(x-\varepsilon_{1} y\right)=x^{2}-y^{2} \in f^{*} \mathbf{m}_{2} \cdot \mathcal{E}_{2}, \\
x y+\gamma x^{2}=f_{2} \in f^{*} \mathbf{m}_{2} .
\end{gathered}
$$

Hence whenever

$$
\operatorname{det}\left(\begin{array}{ccc}
1 & 2 \varepsilon_{1} & 1 \\
1 & 0 & -1 \\
\gamma & 1 & 0
\end{array}\right) \neq 0
$$

then $H^{2}(2,2) \subset f^{*} \mathbf{m}_{2} \cdot \mathcal{E}_{2,2}+\mathbf{m}_{2}^{3} \cdot \mathcal{E}_{2,2}$. The condition 3.3 is not met for $\gamma=0, \varepsilon_{1}=-1$ and $\gamma=2, \varepsilon_{1}=1$. Because by assumption $\gamma \neq 0$, we have to check the remaining case when $\gamma=2, \varepsilon_{1}=1$ but then we have $j^{2}(f)=\left(x+y, x y+2 x^{2}\right)$,

$$
\begin{gathered}
(x+y)=f_{1} \in f^{*} \mathbf{m}_{2} \Rightarrow(x+y)(x-y)=x^{2}-y^{2} \in f^{*} \mathbf{m}_{2} \cdot \mathcal{E}_{2}, \\
(x+y)=f_{1} \in f^{*} \mathbf{m}_{2} \Rightarrow(x+y) x=x^{2}+x y \in f^{*} \mathbf{m}_{2} \cdot \mathcal{E}_{2}, \\
x y+2 x^{2}=f_{2} \in f^{*} \mathbf{m}_{2} .
\end{gathered}
$$

We get a system of 3 linearly independent polynomials. From this system we can obtain all homogeneous terms of degree 2, namely: $x^{2}, x y, y^{2}$. The second condition however is not fulfilled (for $r=2$ ) because for instance $\left(0, y^{3}\right) \notin L \mathcal{B}_{1} \cdot f+\mathbf{m}_{2}^{5} \cdot \mathcal{E}_{2,2}$. However for $r=3$ the second condition is

$$
\mathbf{m}_{2}^{4} \cdot \mathcal{E}_{2,2} \subset L \mathcal{B}_{1} \cdot f+\mathbf{m}_{2}^{6} \cdot \mathcal{E}_{2,2}
$$

and it is fullfilled. This means that every 3 -jet whose 2 -jet is equal to $\left(x+\varepsilon_{1} y, x y+\right.$ $\left.\gamma x^{2}\right)$ is 3 - $\mathcal{B}$-sufficient for $\gamma \neq 0, \frac{1}{2} \varepsilon_{1}, \varepsilon_{1}$. Because the 3 -transversal $T_{3}$ in 3 -jet space to $j^{2}(f)=\left(x+\varepsilon_{1} y, x y+\gamma x^{2}\right)$ is empty, we may deduce that $j^{2}(f)$ is also 2 - $\mathcal{B}$-sufficient. The codimension is 2 and the versal unfolding is

$$
F_{\lambda}(x, y)=\left(\begin{array}{c}
x+\varepsilon_{1} y \\
x y+\gamma x^{2}
\end{array}\right)+\lambda_{1}\left(\begin{array}{l}
0 \\
y
\end{array}\right)+\lambda_{2}\left(\begin{array}{c}
0 \\
y^{2}
\end{array}\right)
$$

At the beginning of this discussion we have assumed that $\gamma \neq 0$, now we consider the remaining exceptional values $\gamma=\frac{1}{2} \varepsilon_{1}$.

A) For the case $\gamma=\frac{1}{2} \varepsilon_{1}$ the complete transversal in 3 -jets is $T_{3}=\left\{\left(0, x^{2} y\right)\right\}$. Then $j^{3}(f) \sim\left(x+\varepsilon_{1} y, x y+\frac{1}{2} \varepsilon_{1} x^{2}+a_{1} x^{2} y\right)$ (see 3.3.3). 
B) For $\gamma=\varepsilon_{1}$ the the complete transversal in 3-jets is $T_{3}=\left\{\left(0, y^{3}\right)\right\}$. Then $j^{3}(f) \sim$ $\left(x+\varepsilon_{1} y, x y+\varepsilon_{1} x^{2}+a_{1} y^{3}\right)$.

B1) For $a_{1}=0, j^{3}(f)=\left(x+\varepsilon_{1} y, x y+\varepsilon_{1} x^{2}\right)($ see 3.3.5).

B2) For $a_{1} \neq 0, j^{3}(f) \sim\left(x+\varepsilon_{1} y, x y+\varepsilon_{1} x^{2}+\varepsilon_{2} y^{3}\right)$ (see 3.3.6).

3.2.5. Case $j^{2}(f)=\left(x+\varepsilon_{1} y, x y\right)$. The complete transversal in 3 -jets is $T=\left\{\left(0, y^{3}\right)\right\}$ so

$$
j^{3}(f) \sim\left(x+\varepsilon_{1} y, x y+a y^{3}\right) .
$$

A) For $a \neq 0, j^{3}(f) \sim\left(x+\varepsilon_{1} y, x y+\varepsilon_{2} y^{3}\right)^{(*)}$ (see 3.3.7).

B) For $a=0, j^{3}(f) \sim\left(x+\varepsilon_{1} y, x y\right)$ (see 3.3.8).

3.2.6. Case $j^{2}(f)=\left(x+\epsilon y, x^{2}\right)$. The 3 -transversal is $T=\left\{\left(0, y^{3}\right)\right\}$ so

$$
j^{3}(f) \sim\left(x+\varepsilon_{1} y, x^{2}+a y^{3}\right) .
$$

A) For $a \neq 0, j^{3}(f) \sim\left(x+\varepsilon_{1} y, x^{2}+\varepsilon_{2} y^{3}\right)^{(*)}$ (see 3.3.9).

B) For $a=0, j^{3}(f) \sim\left(x+\varepsilon_{1} y, x^{2}\right)$ (see 3.3.10).

3.2.7. Case $j^{2}(f)=(x+\epsilon y, 0)$. The 3 -transversal is $T_{3}=\left\{\left(0, x^{2} y\right),\left(0, x y^{2}\right),\left(0, y^{3}\right)\right\}$ so

$$
j^{3}(f) \sim\left(x+\varepsilon_{1} y, a_{1} x^{2} y+a_{2} x y^{2}+a_{3} y^{3}\right) .
$$

In this case however no strata of codimension $\leq 3$ arise.

A) For $a_{1} \neq 0 \vee a_{2} \neq 0 \vee a_{3} \neq 0, \operatorname{codim}_{\mathbb{R}}(f) \geq 5$.

B) For $a_{1}=0 \wedge a_{2}=0 \wedge a_{3}=0, \operatorname{codim}_{\mathbb{R}}(f) \geq 6$.

3.3. 3-jets and higher

3.3.1. Case $j^{3}(f)=\left(x+\varepsilon_{1} y^{3}, x y+\varepsilon_{2} y^{2}\right)^{(*)}$.

$$
\begin{aligned}
& L\left(J^{4} \mathcal{B}_{1}\right) \cdot f=J^{4}\left(\mathcal{E}_{2}\left\langle\left(\begin{array}{c}
x^{2} \\
x^{2} y
\end{array}\right),\left(\begin{array}{c}
x y \\
x y^{2}
\end{array}\right),\left(\begin{array}{c}
3 \varepsilon_{1} x y^{3} \\
x^{2} y+2 \varepsilon_{2} x y^{2}
\end{array}\right),\left(\begin{array}{c}
3 \varepsilon_{1} y^{4} \\
x y^{2}+2 \varepsilon_{2} y^{3}
\end{array}\right)\right\rangle\right) \\
+ & J^{4}\left(f^{*} \mathcal{E}_{2}\left\langle x^{2}+2 \varepsilon_{1} x y^{3}+y^{6}, x^{2} y+\varepsilon_{2} x y^{2}+\varepsilon_{1} x y^{4}+\varepsilon_{1} \varepsilon_{2} y^{5}, x^{2} y^{2}+\varepsilon_{2} x y^{3}+y^{4}\right\rangle\left\{e_{1}, e_{2}\right\}\right) .
\end{aligned}
$$

In this case the 4 -transversal is empty which may suggest that the 3 -jet is sufficient. We prove 3 - $\mathcal{B}$-determinacy using criterion 2.2 with $l=2, r=3$.

The codimension is 3 and the versal unfolding is

$$
F_{\lambda}(x, y)=\left(\begin{array}{c}
x+\varepsilon_{1} y^{3} \\
x y+\varepsilon_{2} y^{2}
\end{array}\right)+\lambda_{1}\left(\begin{array}{l}
y \\
0
\end{array}\right)+\lambda_{2}\left(\begin{array}{l}
0 \\
y
\end{array}\right)+\lambda_{3}\left(\begin{array}{l}
x \\
0
\end{array}\right) .
$$

3.3.2. Case $j^{3}(f)=\left(x, x y+\varepsilon_{2} y^{2}\right)$.

$$
\begin{aligned}
L\left(J^{4} \mathcal{B}_{1}\right) \cdot f= & J^{4}\left(\left\langle\left(\begin{array}{c}
x^{2} \\
x^{2} y
\end{array}\right),\left(\begin{array}{c}
x y \\
x y^{2}
\end{array}\right),\left(\begin{array}{c}
0 \\
x^{2} y+2 \varepsilon_{2} x y^{2}
\end{array}\right),\left(\begin{array}{c}
0 \\
x y^{2}+2 \varepsilon_{2} y^{3}
\end{array}\right)\right\rangle_{\mathcal{E}_{2}}\right) \\
& +\left\langle x^{2}, x^{2} y+\varepsilon_{2} x y^{2}, x^{2} y^{2}+\varepsilon_{2} x y^{3}+y^{4}\right\rangle_{\mathbb{R}}\left\{e_{1}, e_{2}\right\} .
\end{aligned}
$$

The 4-transversal $T_{4}$ is empty. However $j^{4}(f) \sim\left(x, x y+\varepsilon_{2} y^{2}\right)$ is not a sufficient jet because $\mathbf{m}_{2}^{5} . \mathcal{E}_{2,2} \nsubseteq T_{e} \mathcal{B}$.f, in fact

$$
\left(\begin{array}{c}
y^{5} \\
0
\end{array}\right) \notin T_{e} \mathcal{B} \cdot f=\mathcal{E}_{2}\left\{\left(\begin{array}{c}
x \\
x y
\end{array}\right),\left(\begin{array}{c}
0 \\
x y+2 \varepsilon_{2} y^{2}
\end{array}\right)\right\}+f^{*} \mathcal{E}_{2}\left\{e_{1}, e_{2}\right\} .
$$


The next transversal in 5-jets is $T_{5}=\left\{\left(y^{5}, 0\right)\right\}$, so

$$
j^{5}(f) \sim\left(x+a y^{5}, x y+\varepsilon_{2} y^{2}\right) .
$$

A) For $a \neq 0, j^{5}(f) \sim\left(x+\varepsilon_{1} y^{5}, x y+\varepsilon_{2} y^{2}\right)^{(*)}$. Next, using 2.2 with $l=2$ and $r=5$ we prove 5 - $\mathcal{B}$-determinacy. The codimension is 3 and the versal unfolding is

$$
F_{\lambda}(x, y)=\left(\begin{array}{c}
x+\varepsilon_{1} y^{5} \\
x y+\varepsilon_{2} y^{2}
\end{array}\right)+\lambda_{1}\left(\begin{array}{l}
y \\
0
\end{array}\right)+\lambda_{2}\left(\begin{array}{l}
0 \\
y
\end{array}\right)+\lambda_{3}\left(\begin{array}{c}
y^{3} \\
0
\end{array}\right) .
$$

B) For $a=0, j^{5}(f) \sim\left(x, x y+\varepsilon_{2} y^{2}\right)$ but $\operatorname{codim}_{\mathbb{R}}(f) \geq 4$.

3.3.3. Case $j^{3}(f)=\left(x+\varepsilon_{1} y, x y+\frac{1}{2} \varepsilon_{1} x^{2}+a_{1} x^{2} y\right)$.

A) For $a_{1}=0, j^{3}(f)=\left(x+\varepsilon_{1} y, x y+\frac{1}{2} \varepsilon_{1} x^{2}\right)($ see 3.3.4).

B) For $a_{1} \neq 0, j^{3}(f) \sim\left(x+\varepsilon_{1} y, x y+\frac{1}{2} \varepsilon_{1} x^{2}+\varepsilon_{2} x^{2} y\right)^{(*)}$. This case is sufficient. We prove this using determinacy criterion 2.2 for $l=2, r=3$. The versal unfolding is

$$
F_{\lambda}(x, y)=\left(\begin{array}{c}
x+\varepsilon_{1} y \\
x y+\frac{1}{2} \varepsilon_{1} x^{2}+\varepsilon_{2} x^{2} y
\end{array}\right)+\lambda_{1}\left(\begin{array}{l}
0 \\
y
\end{array}\right)+\lambda_{2}\left(\begin{array}{c}
0 \\
x y
\end{array}\right) .
$$

3.3.4. Case $j^{3}(f)=\left(x+\varepsilon_{1} y, x y+\frac{1}{2} \varepsilon_{1} x^{2}\right)$. The complete transversal in 4 -jets is $T_{4}=$ $\left(0, x^{3} y\right)$ so every 4 -jet with 3 -jet equal $\left(x+\varepsilon_{1} y, x y+\frac{1}{2} \varepsilon_{1} x^{2}\right)$ is $J^{4} \mathcal{B}$-equivalent to

$$
j^{4}(f)=\left(x+\varepsilon_{1} y, x y+\frac{1}{2} \varepsilon_{1} x^{2}+a_{1} x^{3} y\right) .
$$

A) For $a_{1}=0, j^{4}(f)=\left(x+\varepsilon_{1} y, x y+\frac{1}{2} \varepsilon_{1} x^{2}\right)$ and $\operatorname{codim}_{\mathbb{R}}(f) \geq 4$.

B) For $a_{1} \neq 0, j^{4}(f) \sim\left(x+\varepsilon_{1} y, x y+\frac{1}{2} \varepsilon_{1} x^{2}+\varepsilon_{2} x^{3} y\right)^{(*)}$. We prove the 4 - $\mathcal{B}$-determinacy using 2.2 with $l=2, r=4$. The codimension is 3 and the versal unfolding is

$$
F_{\lambda}(x, y)=\left(\begin{array}{c}
x+\varepsilon_{1} y \\
x y+\frac{1}{2} \varepsilon_{1} x^{2}+\varepsilon_{2} x^{3} y
\end{array}\right)+\lambda_{1}\left(\begin{array}{l}
0 \\
y
\end{array}\right)+\lambda_{2}\left(\begin{array}{c}
0 \\
x y
\end{array}\right)+\lambda_{3}\left(\begin{array}{c}
0 \\
x^{2} y
\end{array}\right) .
$$

3.3.5. Case $j^{3}(f)=\left(x+\varepsilon_{1} y, x y+\varepsilon_{1} x^{2}\right)$. The complete transversal in 4 -jets is $T_{4}=\left(0, y^{4}\right)$ SO

$$
j^{4}(f) \sim\left(x+\varepsilon_{1} y, x y+\varepsilon_{1} x^{2}+a_{1} y^{4}\right) .
$$

A) For $a_{1}=0, j^{4}(f)=\left(x+\varepsilon_{1} y, x y+\varepsilon_{1} x^{2}\right), \operatorname{codim}_{\mathbb{R}}(f) \geq 4$.

B) For $a_{1} \neq 0, j^{4}(f) \sim\left(x+\varepsilon_{1} y, x y+\varepsilon_{1} x^{2}+\varepsilon_{2} y^{4}\right)$. The next transversal is $T_{5}=\left(0, y^{5}\right)$ however using Mather's Lemma we may reduce the term $\left(0, y^{5}\right)$. The transversal in 6 -jets is $T_{6}=\left(0, y^{6}\right)$ so $j^{6}(f) \sim\left(x+\varepsilon_{1} y, x y+\varepsilon_{1} x^{2}+\varepsilon_{2} y^{4}+a_{1} y^{6}\right)$. In such a case we get $\operatorname{codim}_{\mathbb{R}}(f) \geq 4$.

3.3.6. Case $j^{3}(f)=\left(x+\varepsilon_{1} y, x y+\varepsilon_{1} x^{2}+\varepsilon_{2} y^{3}\right)^{(*)}$. The complete transversal in 4 -jets is $T_{4}=\left(0, y^{4}\right)$ so every 4 -jet whose 3 -jet is equal to $\left(x+\varepsilon_{1} y, x y+\varepsilon_{1} x^{2}+\varepsilon_{2} y^{3}\right)$ is $J^{4} \mathcal{B}$-equivalent to

$$
j^{4}(f) \sim\left(x+\varepsilon_{1} y, x y+\varepsilon_{1} x^{2}+\varepsilon_{2} y^{3}+a_{1} y^{4}\right) .
$$

Then we use Mather's Lemma to show that all 4-jets for all values of the parameter $a_{1}$ are actually contained in a single orbit of the action of $J^{4} \mathcal{B}$. We simplify the transversal further to $T_{4}=\emptyset$. We may expect that $j^{3}(f)=\left(x+\varepsilon_{1} y, x y+\varepsilon_{1} x^{2}+\varepsilon_{2} y^{3}\right)$ is sufficient. We apply the determinacy criterion for $l=2, r=3$, but it turns out that the second condition fails to hold for $r=3$ because in the inclusion

$$
\mathbf{m}_{2}^{4} \cdot \mathcal{E}_{2,2} \subset L \mathcal{B}_{1} \cdot f+\mathbf{m}_{2}^{6} \cdot \mathcal{E}_{2,2}
$$


we have missing term $\left(0, y^{4}\right)$. In this situation we check the 5 -transversal which is also empty and we try to prove 4-determinacy for the 4-jet $j^{4}(f)=\left(x+\varepsilon_{1} y, x y+\varepsilon_{1} x^{2}+\varepsilon_{2} y^{3}\right)$ using 2.2 for $l=2, r=4$. This time both conditions hold, which means that our 4 -jet is sufficient. We recall however that 4 -transversal $T_{4}=\emptyset$ so we may deduce that the 3 -jet $j^{3}(f)=\left(x+\varepsilon_{1} y, x y+\varepsilon_{1} x^{2}+\varepsilon_{2} y^{3}\right)$ is also sufficient. The codimension is 2 and the versal unfolding is

$$
F_{\lambda}(x, y)=\left(\begin{array}{c}
x+\varepsilon_{1} y \\
x y+\varepsilon_{1} x^{2}+\varepsilon_{2} y^{3}
\end{array}\right)+\lambda_{1}\left(\begin{array}{l}
0 \\
y
\end{array}\right)+\lambda_{2}\left(\begin{array}{c}
0 \\
y^{2}
\end{array}\right) .
$$

3.3.7. Case $j^{3}(f)=\left(x+\varepsilon_{1} y, x y+\varepsilon_{2} y^{3}\right)^{(*)}$. The complete transversal in 4 -jet space is empty. Next, we prove 3 - $\mathcal{B}$-determinacy using 2.2 with $l=2, r=3$. For the first condition it is enough to observe that

$$
\mathbf{m}_{2}^{3} \subset \mathcal{E}_{2}\left\langle x+\varepsilon_{1} y, x y+\varepsilon_{2} y^{3}\right\rangle \bmod \mathbf{m}_{2}^{3} .
$$

For the second condition we work modulo $\mathbf{m}_{2}^{6} \cdot \mathcal{E}_{2,2}$ so it is enough to show that all homogeneous terms of degree 4 and 5 are contained in $L \mathcal{B}_{1}$ tangent space modulo $\mathbf{m}_{2}^{6} \cdot \mathcal{E}_{2,2}$.

$$
H^{4}(2,2) \cup H^{5}(2,2) \subset L \mathcal{B}_{1} . f+\mathbf{m}_{2}^{6} \cdot \mathcal{E}_{2,2} .
$$

The codimension is 2 and the versal unfolding is

$$
F_{\lambda}(x, y)=\left(\begin{array}{c}
x+\varepsilon_{1} y \\
x y+\varepsilon_{2} y^{3}
\end{array}\right)+\lambda_{1}\left(\begin{array}{l}
0 \\
y
\end{array}\right)+\lambda_{2}\left(\begin{array}{c}
0 \\
y^{2}
\end{array}\right) .
$$

3.3.8. Case $j^{3}(f)=\left(x+\varepsilon_{1} y, x y\right)$. The 4 -transversal is $T_{4}=\left\{\left(0, y^{4}\right)\right\}$ so

$$
j^{4}(f) \sim\left(x+\varepsilon_{1} y, x y+a y^{4}\right) .
$$

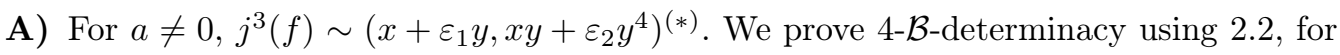
$l=2, r=4$. The codimension is 3 and the versal unfolding is

$$
F_{\lambda}(x, y)=\left(\begin{array}{c}
x+\varepsilon_{1} y \\
x y+\varepsilon_{2} y^{4}
\end{array}\right)+\lambda_{1}\left(\begin{array}{l}
0 \\
y
\end{array}\right)+\lambda_{2}\left(\begin{array}{c}
0 \\
y^{2}
\end{array}\right)+\lambda_{3}\left(\begin{array}{c}
0 \\
y^{3}
\end{array}\right) \text {. }
$$

B) For $a=0, j^{4}(f)=\left(x+\varepsilon_{1} y, x y\right)$ and $\operatorname{codim}_{\mathbb{R}}(f) \geq 4$.

3.3.9. Case $j^{3}(f)=\left(x+\varepsilon_{1} y, x^{2}+\varepsilon_{2} y^{3}\right)^{(*)}$. The 4 -transversal is empty. We prove $3-\mathcal{B}$ determinacy using 2.2 with $l=2, r=3$. The codimension is 2 and the versal unfolding is

$$
F_{\lambda}(x, y)=\left(\begin{array}{c}
x+\varepsilon_{1} y \\
x^{2}+\varepsilon_{2} y^{3}
\end{array}\right)+\lambda_{1}\left(\begin{array}{l}
0 \\
x
\end{array}\right)+\lambda_{2}\left(\begin{array}{c}
0 \\
x y
\end{array}\right) .
$$

3.3.10. Case $j^{3}(f)=\left(x+\varepsilon_{1} y, x^{2}\right)$. The 4 -transveral is $T=\left\{\left(0, y^{4}\right)\right\}$ so

$$
j^{4}(f) \sim\left(x+\varepsilon_{1} y, x^{2}+a y^{4}\right) .
$$

A) For $a \neq 0, j^{4}(f) \sim\left(x+\varepsilon_{1} y, x^{2}+\varepsilon_{2} y^{4}\right)^{(*)}$. This germ is sufficient. We prove 4$\mathcal{B}$-determinacy using 2.2 with $l=2 i r=4$. The codimension is 3 and the versal unfolding is

$$
F_{\lambda}(x, y)=\left(\begin{array}{c}
x+\varepsilon_{1} y \\
x^{2}+\varepsilon_{2} y^{4}
\end{array}\right)+\lambda_{1}\left(\begin{array}{l}
0 \\
y
\end{array}\right)+\lambda_{2}\left(\begin{array}{c}
0 \\
y^{2}
\end{array}\right)+\lambda_{3}\left(\begin{array}{c}
0 \\
y^{3}
\end{array}\right) .
$$

B) For $a=0, j^{4}(f)=\left(x+\varepsilon_{1} y, x^{2}\right)$, however $\operatorname{codim}_{\mathbb{R}}(f) \geq 4$. 
4. Classification results. In this section we present results of classification of the mapgerms. We restrict to germs of corank $\leq 1$ and codimension $\leq 3$. Symmetry of the problem allows us to list only forms originating from the 1-jet $(x, 0)$. A full list contains additionally all forms obtained by interchanging $x \leftrightarrow y$.

Table 1. Classification results

\begin{tabular}{llc}
\hline Id. & Normal form & $\mathcal{B}$-codimension of orbit \\
\hline I & $(x, y)$ & 0 \\
II & $\left(x+\varepsilon_{1} y^{3}, x y+\varepsilon_{2} y^{2}\right)$ & 3 \\
III & $\left(x+\varepsilon_{1} y^{5}, x y+\varepsilon_{2} y^{2}\right)$ & 3 \\
IV & $\left(x+\varepsilon_{1} y, x y+\varepsilon_{2} y^{3}\right)$ & 2 \\
V & $\left(x+\varepsilon_{1} y, x y+\varepsilon_{2} y^{4}\right)$ & 3 \\
VI & $\left(x+\varepsilon_{1} y, x^{2}+\varepsilon_{2} y^{3}\right)$ & 2 \\
VII & $\left(x+\varepsilon_{1} y, x^{2}+\varepsilon_{2} y^{4}\right)$ & 3 \\
VIII & $\left(x+\varepsilon_{1} y, x y+\gamma x^{2}\right)$ & 2 \\
IX & $\left(x+\varepsilon_{1} y, x y+\frac{1}{2} \varepsilon_{1} x^{2}+\varepsilon_{2} x^{2} y\right)$ & 2 \\
X & $\left(x+\varepsilon_{1} y, x y+\frac{1}{2} \varepsilon_{1} x^{2}+\varepsilon_{2} x^{3} y\right)$ & 2 \\
XI & $\left(x+\varepsilon_{1} y, x y+\varepsilon_{1} x^{2}+\varepsilon_{2} y^{3}\right)$ & 2 \\
\hline
\end{tabular}

The only stable form in the above list is the submersion $(x, y)$. Other forms are not $\mathcal{B}$-stable.

THEOREM 4.1 (List of universal unfoldings). Universal unfoldings for the germs from table 1 are given in the list below.

$$
\begin{aligned}
& \text { II } \quad F_{\lambda}(x, y)=\left(\begin{array}{c}
x+\varepsilon_{1} y^{3} \\
x y+\varepsilon_{2} y^{2}
\end{array}\right)+\lambda_{1}\left(\begin{array}{l}
y \\
0
\end{array}\right)+\lambda_{2}\left(\begin{array}{l}
0 \\
y
\end{array}\right)+\lambda_{3}\left(\begin{array}{c}
x \\
0
\end{array}\right) \\
& \text { III } \quad F_{\lambda}(x, y)=\left(\begin{array}{c}
x+\varepsilon_{1} y^{5} \\
x y+\varepsilon_{2} y^{2}
\end{array}\right)+\lambda_{1}\left(\begin{array}{l}
y \\
0
\end{array}\right)+\lambda_{2}\left(\begin{array}{l}
0 \\
y
\end{array}\right)+\lambda_{3}\left(\begin{array}{c}
y^{3} \\
0
\end{array}\right) \\
& I V \quad F_{\lambda}(x, y)=\left(\begin{array}{c}
x+\varepsilon_{1} y \\
x y+\varepsilon_{2} y^{3}
\end{array}\right)+\lambda_{1}\left(\begin{array}{l}
0 \\
y
\end{array}\right)+\lambda_{2}\left(\begin{array}{c}
0 \\
y^{2}
\end{array}\right) \\
& V \quad F_{\lambda}(x, y)=\left(\begin{array}{c}
x+\varepsilon_{1} y \\
x y+\varepsilon_{2} y^{4}
\end{array}\right)+\lambda_{1}\left(\begin{array}{l}
0 \\
y
\end{array}\right)+\lambda_{2}\left(\begin{array}{c}
0 \\
y^{2}
\end{array}\right)+\lambda_{3}\left(\begin{array}{c}
0 \\
y^{3}
\end{array}\right) \\
& V I \quad F_{\lambda}(x, y)=\left(\begin{array}{c}
x+\varepsilon_{1} y \\
x^{2}+\varepsilon_{2} y^{3}
\end{array}\right)+\lambda_{1}\left(\begin{array}{l}
0 \\
x
\end{array}\right)+\lambda_{2}\left(\begin{array}{c}
0 \\
x y
\end{array}\right) \\
& V I I \quad F_{\lambda}(x, y)=\left(\begin{array}{c}
x+\varepsilon_{1} y \\
x^{2}+\varepsilon_{2} y^{4}
\end{array}\right)+\lambda_{1}\left(\begin{array}{l}
0 \\
y
\end{array}\right)+\lambda_{2}\left(\begin{array}{c}
0 \\
y^{2}
\end{array}\right)+\lambda_{3}\left(\begin{array}{c}
0 \\
y^{3}
\end{array}\right) \\
& \text { VIII } \quad F_{\lambda}(x, y)=\left(\begin{array}{c}
x+\varepsilon_{1} y \\
x y+\gamma x^{2}
\end{array}\right)+\lambda_{1}\left(\begin{array}{c}
0 \\
y
\end{array}\right)+\lambda_{2}\left(\begin{array}{c}
0 \\
y^{2}
\end{array}\right) \quad \text { for } \gamma \neq 0, \frac{1}{2} \varepsilon_{1}, \varepsilon_{1} \\
& I X \quad F_{\lambda}(x, y)=\left(\begin{array}{c}
x+\varepsilon_{1} y \\
x y+\frac{1}{2} \varepsilon_{1} x^{2}+\varepsilon_{2} x^{2} y
\end{array}\right)+\lambda_{1}\left(\begin{array}{c}
0 \\
y
\end{array}\right)+\lambda_{2}\left(\begin{array}{c}
0 \\
x y
\end{array}\right) \\
& X \quad F_{\lambda}(x, y)=\left(\begin{array}{c}
x+\varepsilon_{1} y \\
x y+\frac{1}{2} \varepsilon_{1} x^{2}+\varepsilon_{2} x^{3} y
\end{array}\right)+\lambda_{1}\left(\begin{array}{c}
0 \\
y
\end{array}\right)+\lambda_{2}\left(\begin{array}{c}
0 \\
x y
\end{array}\right)+\lambda_{3}\left(\begin{array}{c}
0 \\
x^{2} y
\end{array}\right) \\
& X I \quad F_{\lambda}(x, y)=\left(\begin{array}{c}
x+\varepsilon_{1} y \\
x y+\varepsilon_{1} x^{2}+\varepsilon_{2} y^{3}
\end{array}\right)+\lambda_{1}\left(\begin{array}{c}
0 \\
y
\end{array}\right)+\lambda_{2}\left(\begin{array}{c}
0 \\
y^{2}
\end{array}\right)
\end{aligned}
$$


5. Geometric realizations. Versal deformations of germs in low codimension $(\leq 2)$ are realized by 2-parameter families of projections of surfaces with singular boundary. Let

$$
i: \mathbb{R}^{2} \hookrightarrow \mathbb{R}^{3}, \quad i(x, y)=(X(x, y), Y(x, y), Z(x, y))
$$

be a smooth immersion so the image of $i$ might be a piece of smooth surface in $\mathbb{R}^{3}$. Next, we consider a family of linear projections $\mathbb{R}^{3} \rightarrow \mathbb{R}^{2}$. The 2-dim family of directions of projection from the point $(0,0,1)$ onto the subspace $z=0$ may be parametrized as

$$
\pi_{\lambda, \mu}(x, y, z)=(x+\lambda z, y+\mu z) .
$$

Composing the immersion with the family of linear projections we get a family of maps from $\mathbb{R}^{2}$ to $\mathbb{R}^{2}$,

$$
i \circ \pi_{\lambda, \mu}(x, y)=(X(x, y)+\lambda Z(x, y), Y(x, y)+\mu Z(x, y)) .
$$

This 2-parameter family of maps may be considered as a special kind of a versal deformation of a map germ $\mathbb{R}^{2} \rightarrow \mathbb{R}^{2}$ with the same unfolding functions namely $Z(x, y)$.

Now given a universal deformation of a singular map germ in low codimension which is actually symmetric

$$
F_{\lambda}(x, y)=F+\lambda_{1} f_{1}+\lambda_{2} f_{2}=\left(X(x, y)+\lambda_{1} Z(x, y), Y(x, y)+\lambda_{2} Z(x, y)\right)
$$

we may recover immersion $i$ of a particular surface in $\mathbb{R}^{3}$ whose projection from the point $(0,0,1)$ onto the $z=0$ plane realizes singularity of $F$ and all singularities near to $F$ in the versal deformation $F_{\lambda}$.

Not all germs admit symmetric deformations. We may have to transform the germ to its $\mathcal{B}$-equivalent germ which admits symmetrical unfolding. Such is the case of Form II.

5.1. List of universal unfoldings in codimension $\leq 2$

$$
\begin{array}{rlrl}
I & F(x, y) & =\left(\begin{array}{l}
x \\
y
\end{array}\right) \\
I V & F_{\lambda}(x, y) & =\left(\begin{array}{c}
x+\varepsilon_{1} y \\
x y+\varepsilon_{2} y^{3}
\end{array}\right)+\lambda_{1}\left(\begin{array}{l}
0 \\
y
\end{array}\right)+\lambda_{2}\left(\begin{array}{c}
0 \\
y^{2}
\end{array}\right) \\
V I & F_{\lambda}(x, y) & =\left(\begin{array}{c}
x+\varepsilon_{1} y \\
x^{2}+\varepsilon_{2} y^{3}
\end{array}\right)+\lambda_{1}\left(\begin{array}{l}
0 \\
x
\end{array}\right)+\lambda_{2}\left(\begin{array}{c}
0 \\
x y
\end{array}\right) \\
V I I I & F_{\lambda}(x, y) & =\left(\begin{array}{c}
x+\varepsilon_{1} y \\
x y+\gamma x^{2}
\end{array}\right)+\lambda_{1}\left(\begin{array}{l}
0 \\
y
\end{array}\right)+\lambda_{2}\left(\begin{array}{c}
0 \\
y^{2}
\end{array}\right) \\
I X & F_{\lambda}(x, y) & =\left(\begin{array}{c}
x+\varepsilon_{1} y \\
x y+\frac{1}{2} \varepsilon_{1} x^{2}+\varepsilon_{2} x^{2} y
\end{array}\right)+\lambda_{1}\left(\begin{array}{c}
0 \\
y
\end{array}\right)+\lambda_{2}\left(\begin{array}{c}
0 \\
x y
\end{array}\right) \\
X I & F_{\lambda}(x, y) & =\left(\begin{array}{c}
x+\varepsilon_{1} y \\
x y+\varepsilon_{1} x^{2}+\varepsilon_{2} y^{3}
\end{array}\right)+\lambda_{1}, \varepsilon_{1}\left(\begin{array}{c}
0 \\
y
\end{array}\right)+\lambda_{2}\left(\begin{array}{c}
0 \\
y^{2}
\end{array}\right)
\end{array}
$$


5.2. Symmetric deformations

$$
\begin{aligned}
I V & F_{\lambda}(x, y) & =\left(\begin{array}{c}
x+\varepsilon_{1} y \\
\varepsilon_{2} y^{3}-x^{2}-y^{2}+\left(1-2 \varepsilon_{1}\right) x y
\end{array}\right)+\lambda_{1}\left(\begin{array}{l}
0 \\
y
\end{array}\right)+\lambda_{2}\left(\begin{array}{l}
y \\
0
\end{array}\right) \\
V I & F_{\lambda}(x, y) & =\left(\begin{array}{c}
x+\varepsilon_{1} y \\
x^{2}+\varepsilon_{2} y^{3}
\end{array}\right)+\lambda_{1}\left(\begin{array}{l}
0 \\
y
\end{array}\right)+\lambda_{2}\left(\begin{array}{l}
y \\
0
\end{array}\right) \\
V I I I & F_{\lambda}(x, y) & =\left(\begin{array}{c}
x+\varepsilon_{1} y \\
x y+\gamma x^{2}
\end{array}\right)+\lambda_{1}\left(\begin{array}{l}
0 \\
y
\end{array}\right)+\lambda_{2}\left(\begin{array}{l}
y \\
0
\end{array}\right) \text { for } \gamma \neq 0, \frac{1}{2} \varepsilon_{1}, \varepsilon_{1} \\
I X & F_{\lambda}(x, y) & =\left(\begin{array}{c}
x+\varepsilon_{1} y \\
x y+\frac{1}{2} \varepsilon_{1} x^{2}+\varepsilon_{2} x^{2} y
\end{array}\right)+\lambda_{1}\left(\begin{array}{l}
0 \\
y
\end{array}\right)+\lambda_{2}\left(\begin{array}{l}
y \\
0
\end{array}\right) \\
X I & F_{\lambda}(x, y) & =\left(\begin{array}{c}
x+\varepsilon_{1} y \\
x y+\varepsilon_{1} x^{2}+\varepsilon_{2} y^{3}
\end{array}\right)+\lambda_{1}\left(\begin{array}{l}
0 \\
y
\end{array}\right)+\lambda_{2}\left(\begin{array}{l}
y \\
0
\end{array}\right)
\end{aligned}
$$

5.3. Pictures of geometric realizations. Below we present pictures of the geometric realizations. For each form we give a picture of the singular set of the mapping $F$ and the kernel line of $d F(0)$. The kernel lines are dashed. In the next picture there is the image of the singular boundary which is drawn black bold and the image of the singular set drawn black. We picture them for the map $F$ and for selected maps from the versal deformation corresponding to the points on the unit sphere in the $\lambda_{1}, \lambda_{2}$ parameter space. Parts of the singular set curve and the image of the singular set which correspond to the part $\{x<0, y<0\}$ in the source are drawn light gray. All plots are made for $\varepsilon_{1}, \varepsilon_{2}=1$.

\subsubsection{Form IV}

$$
F_{\lambda}(x, y)=\left(\begin{array}{c}
x+\varepsilon_{1} y \\
\varepsilon_{2} y^{3}-x^{2}-y^{2}+\left(1-2 \varepsilon_{1}\right) x y
\end{array}\right)+\lambda_{1}\left(\begin{array}{l}
0 \\
y
\end{array}\right)+\lambda_{2}\left(\begin{array}{l}
y \\
0
\end{array}\right)
$$

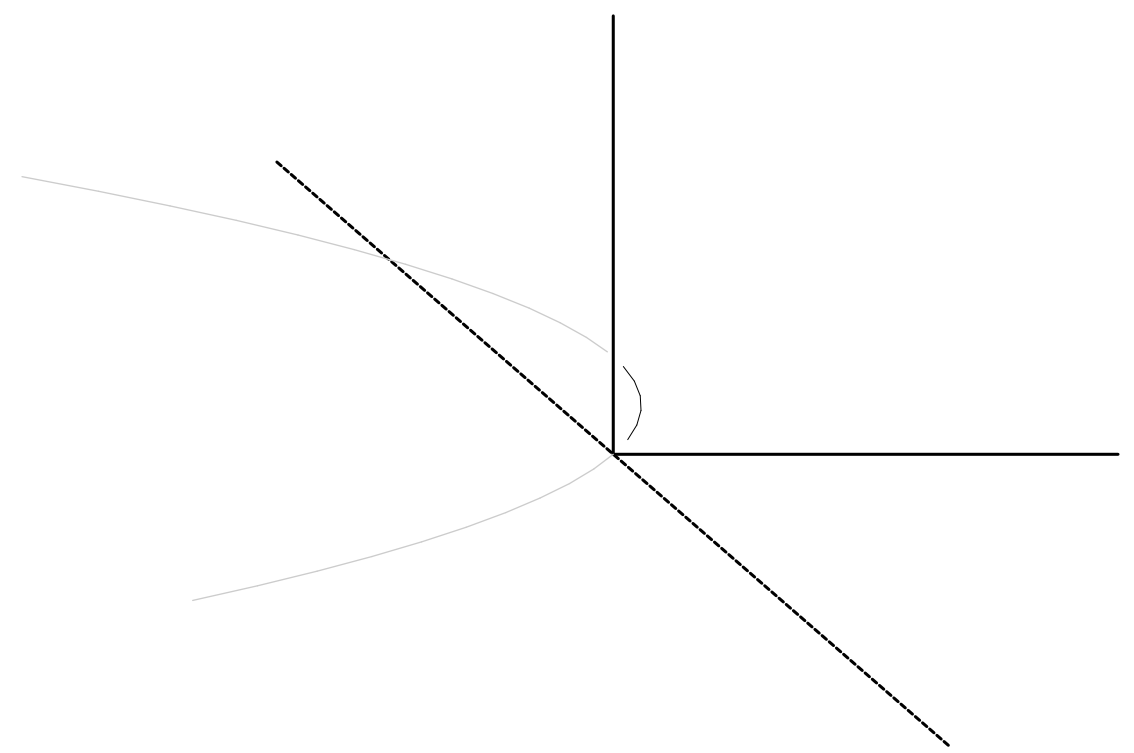

Fig. 1. Critical set and the kernel line: Form IV 

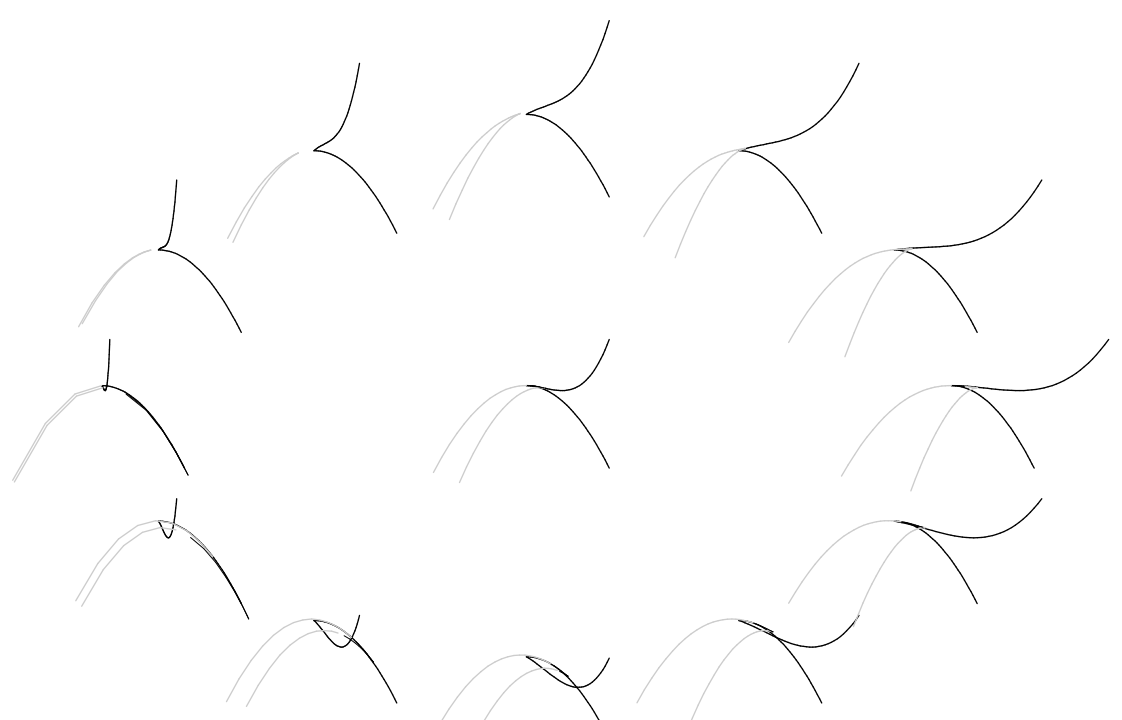

Fig. 2. Image of the boundary and critical loci: Form IV

\subsubsection{Form VI}

$$
F_{\lambda}(x, y)=\left(\begin{array}{c}
x+\varepsilon_{1} y \\
x^{2}+\varepsilon_{2} y^{3}
\end{array}\right)+\lambda_{1}\left(\begin{array}{l}
0 \\
y
\end{array}\right)+\lambda_{2}\left(\begin{array}{l}
y \\
0
\end{array}\right)
$$

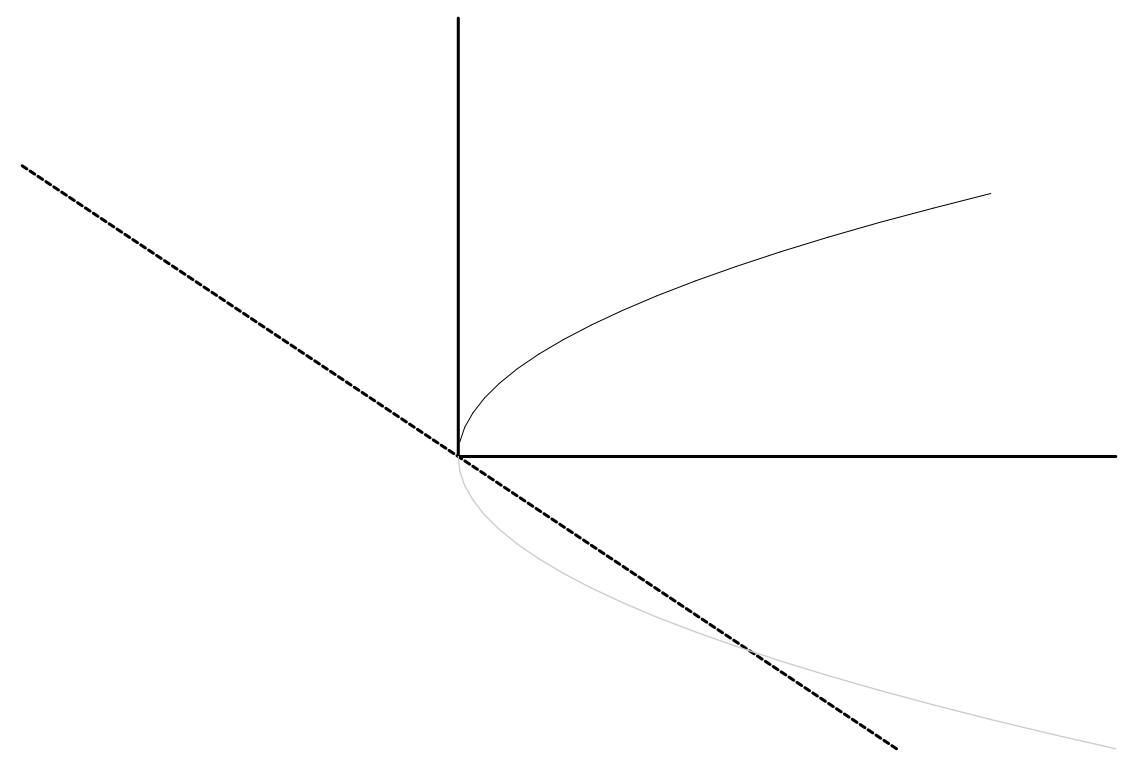

Fig. 3. Critical set and the kernel line: Form VI 

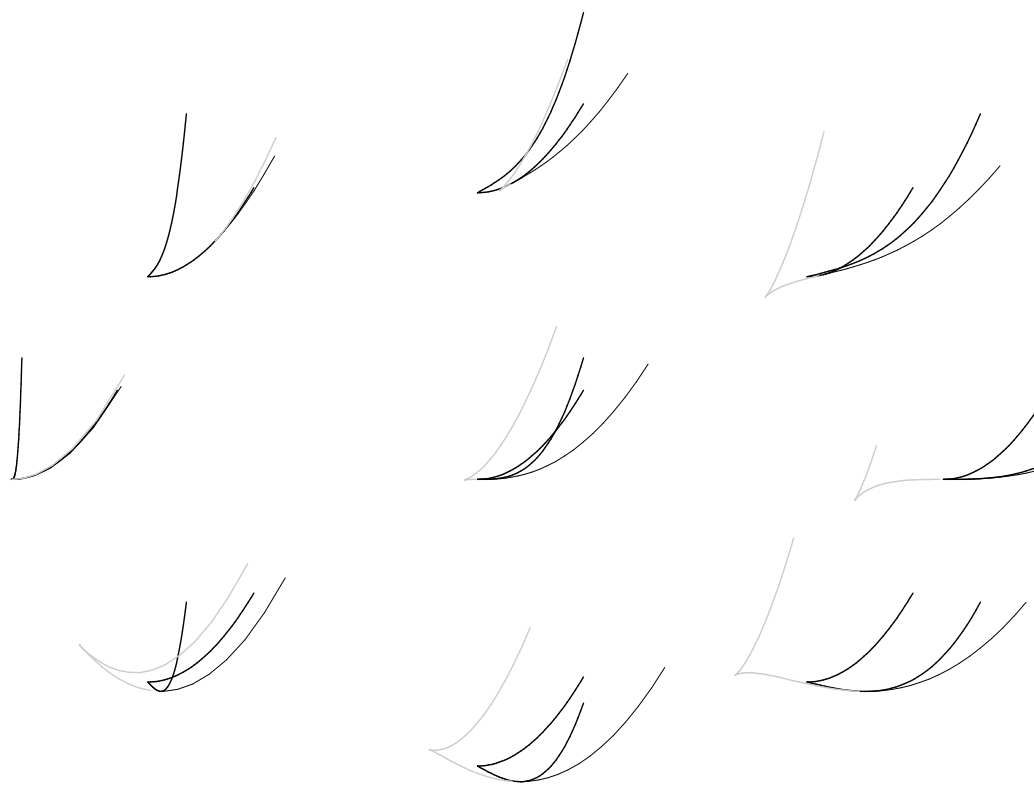

Fig. 4. Image of the boundary and critical loci: Form VI

\subsubsection{Form VIII}

$$
F_{\lambda}(x, y)=\left(\begin{array}{c}
x+\varepsilon_{1} y \\
x y+\gamma x^{2}
\end{array}\right)+\lambda_{1}\left(\begin{array}{l}
0 \\
y
\end{array}\right)+\lambda_{2}\left(\begin{array}{l}
y \\
0
\end{array}\right), \quad \text { example plots for } \gamma=2
$$

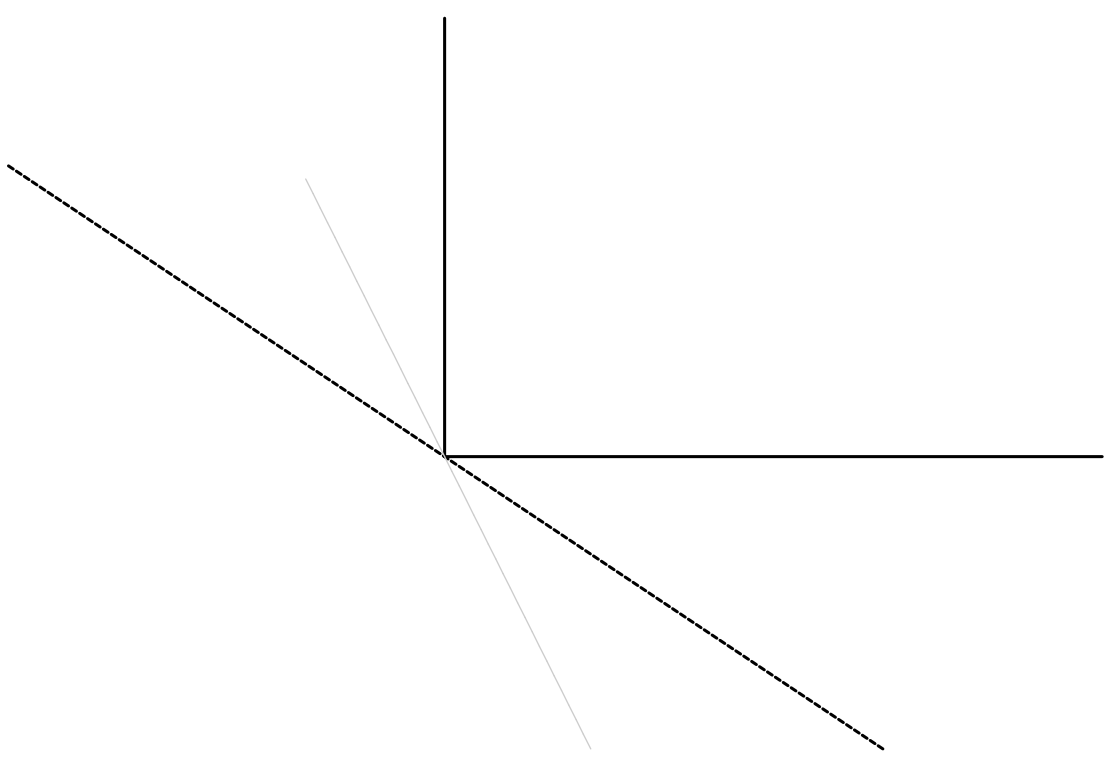

Fig. 5. Critical set and the kernel line: Form VIII 

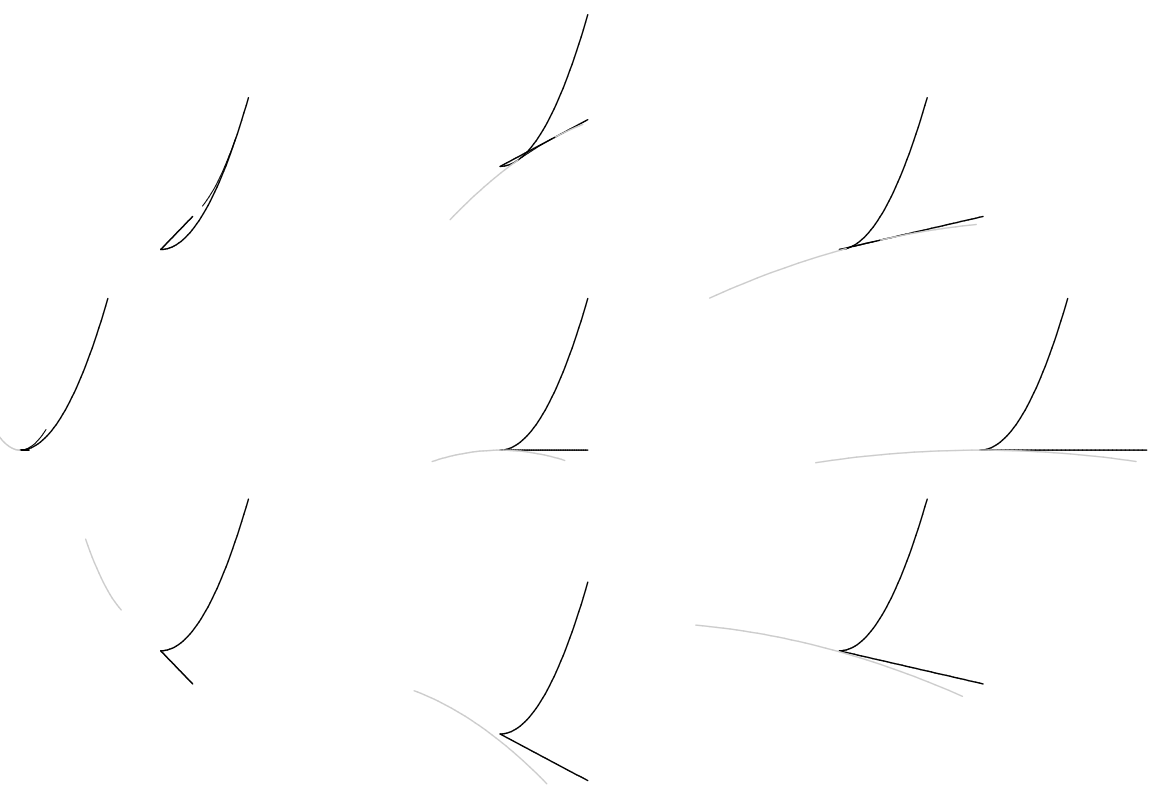

Fig. 6. Image of the boundary and critical loci: Form VIII

\subsubsection{Form $I X$}

$$
F_{\lambda}(x, y)=\left(\begin{array}{c}
x+\varepsilon_{1} y \\
x y+\frac{1}{2} \varepsilon_{1} x^{2}+\varepsilon_{2} x^{2} y
\end{array}\right)+\lambda_{1}\left(\begin{array}{l}
0 \\
y
\end{array}\right)+\lambda_{2}\left(\begin{array}{l}
y \\
0
\end{array}\right)
$$

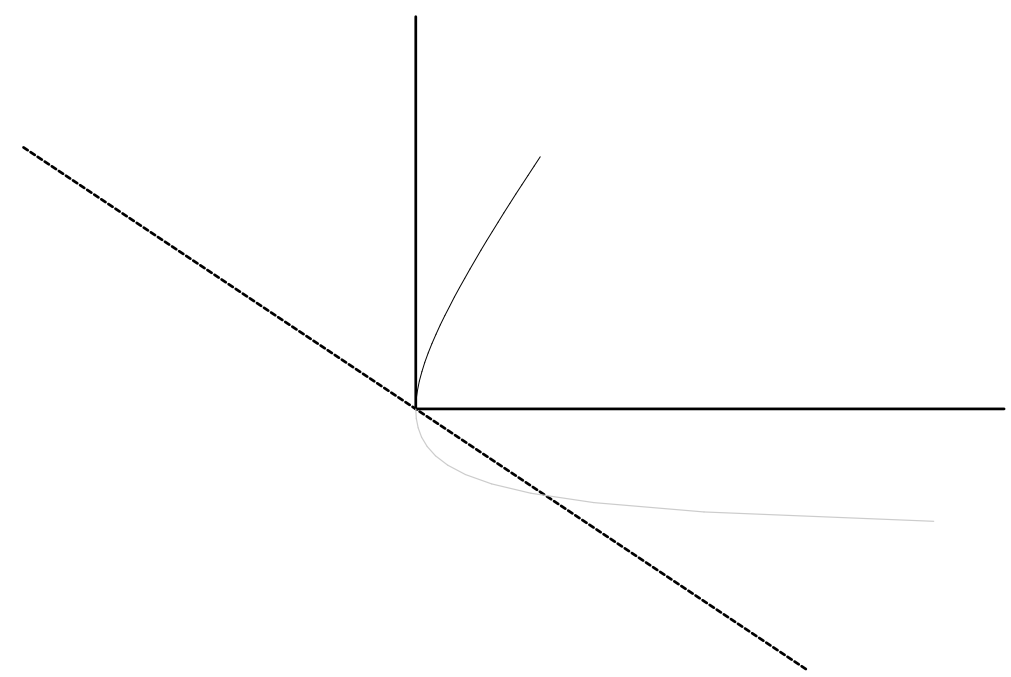

Fig. 7. Critical set and the kernel line: Form IX 

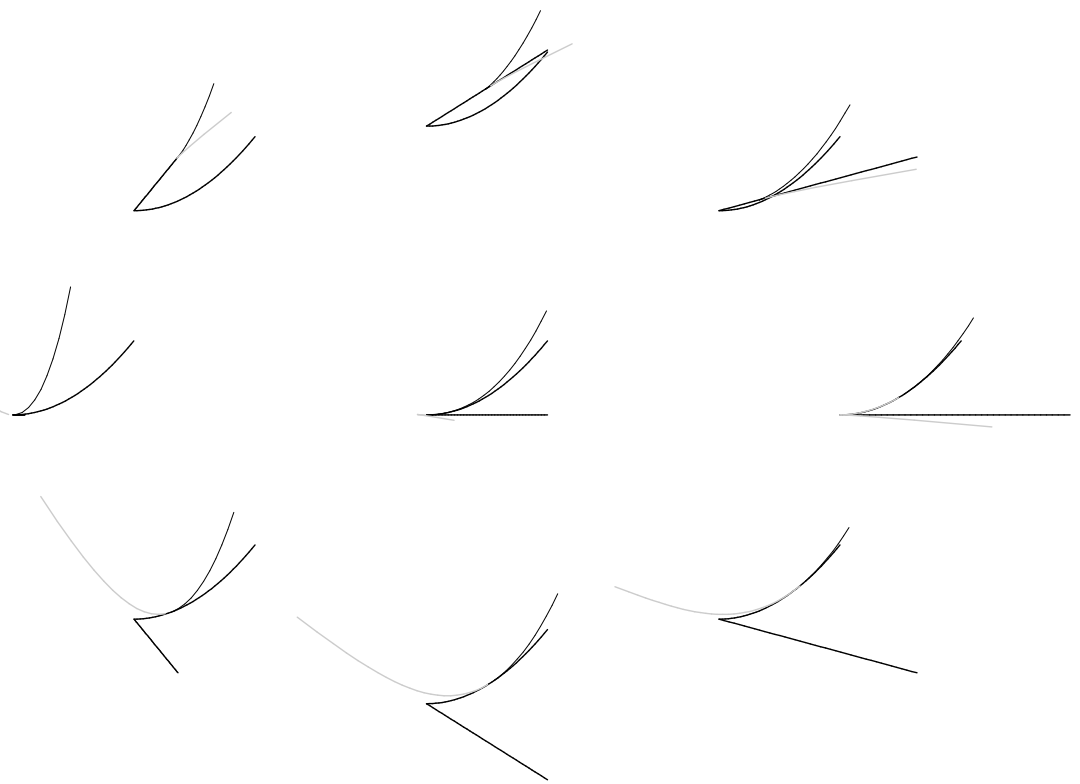

Fig. 8. Image of the boundary and critical loci: Form IX

\subsubsection{Form $X I$}

$$
F_{\lambda}(x, y)=\left(\begin{array}{c}
x+\varepsilon_{1} y \\
x y+\varepsilon_{1} x^{2}+\varepsilon_{2} y^{3}
\end{array}\right)+\lambda_{1}\left(\begin{array}{l}
0 \\
y
\end{array}\right)+\lambda_{2}\left(\begin{array}{l}
y \\
0
\end{array}\right)
$$

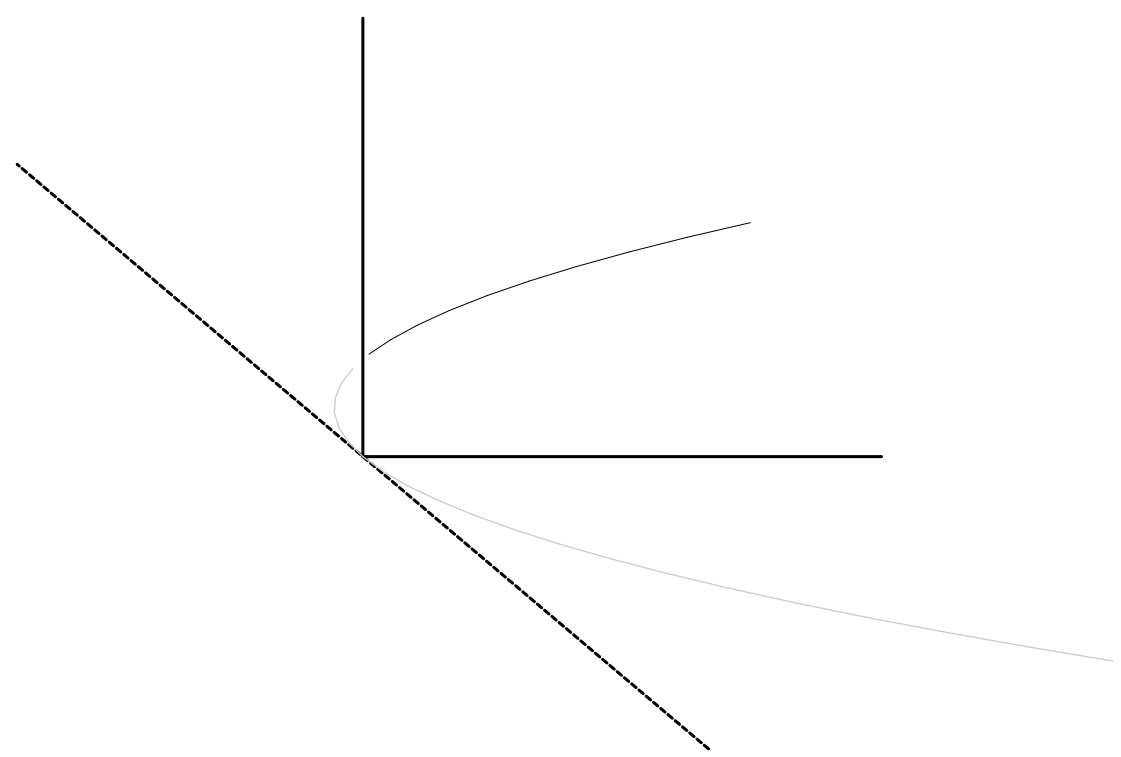

Fig. 9. Critical set and the kernel line: Form XI 

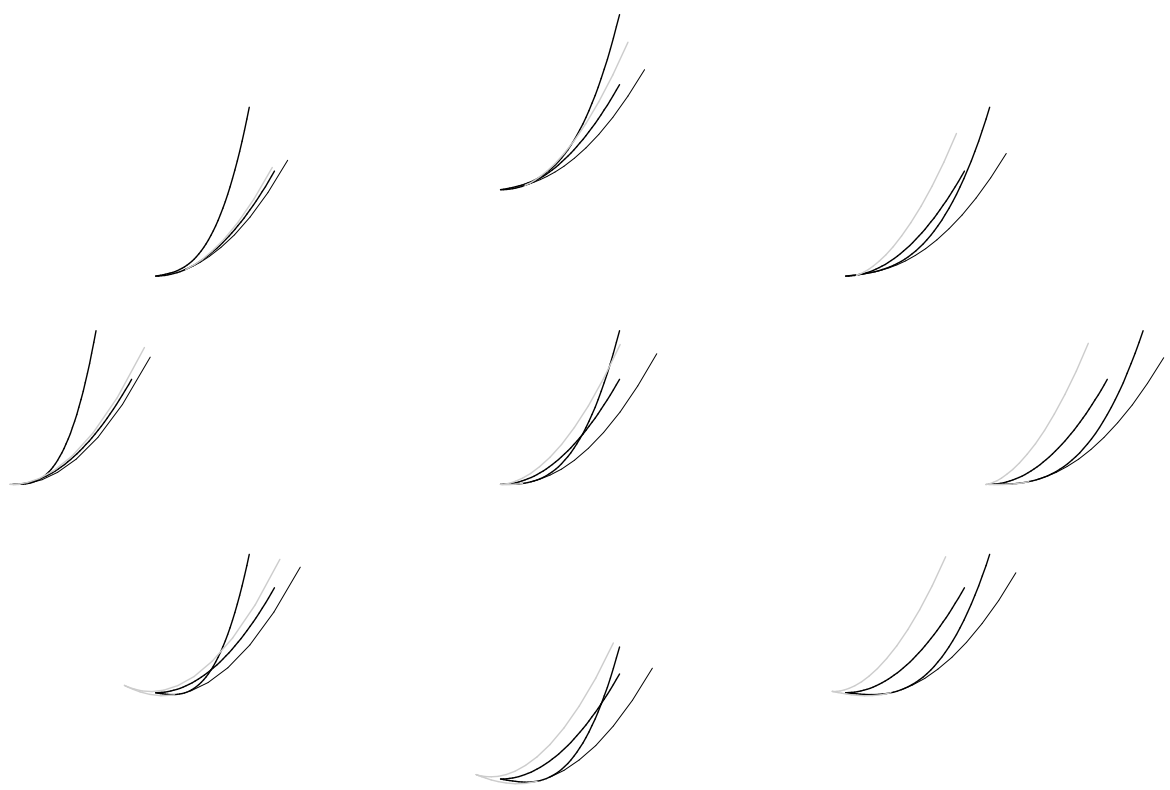

Fig. 10. Image of the boundary and critical loci: Form XI

6. Final remarks. It is easy to see that the subgroup of diffeomorphisms preserving two transversally intersecting lines in $\mathbb{R}^{2}$ embeds canonically into the subgroup preserving only one line. This fact implies that $\mathcal{B}$ is a subgroup of the group considered by Bruce and Giblin in [2]. This explains why each orbit in their work splits into suborbits in view of our classification. Notice that this may be observed when comparing trees of inductive classifications.

The first step of classification of projections of surfaces with singular boundary is completed. It's worth asking about structure of singularities of projections of surfaces with more singular boundary. For instance $\mathcal{A}$-classification of germs of singular curves $g:(\mathbb{R}, 0) \rightarrow\left(\mathbb{R}^{2}, 0\right)$ is known. We may then ask for a classification of projections of surfaces whose boundary is locally diffeomorphic to the germ of a singular curve $g$.

\section{References}

[1] V. I. Arnold, Indices of singular points of 1-forms on a manifold with boundary, convolution of invariants of reflection groups, and singular projections of smooth surfaces, Russian Math. Surveys 34 (1979), 1-42.

[2] J. W. Bruce and P. J. Giblin, Projections of surfaces with boundary, Proc. London Math. Soc. (3) 60 (1990), 392-416.

[3] J. W. Bruce, N. P. Kirk and A. A. du Plessis, Complete transversals and the classification of singularities, Nonlinearity 10 (1997), 253-275.

[4] J. W. Bruce, A. A. du Plessis and C. T. C. Wall, Determinacy and unipotency, Invent. Math. 88 (1987), 521-554 
[5] J. W. Bruce, Classifications in singularity theory and their applications, in: D. Siersma et al. (eds.), New Developments in Singularity Theory, Kluwer, 2001, 3-33.

[6] J. N. Damon, The unfolding and determinacy theorems for subgroups of $\mathcal{A}$ and $\mathbb{K}$, Mem. Amer. Math. Soc. 306 (1984),

[7] M. Golubitsky and V. Guillemin, Stable Mappings and Their Singularities, SpringerVerlag, New York, 1976.

[8] V. Goryunov, Projections of generic surfaces with boundary, in: Theory of Singularities and its Applications, V. I. Arnold (ed.), Advances in Soviet Math., AMS, Providence, RI, 1990, 157-200.

[9] S. Janeczko, Wybrane zagadnienia teorii katastrof, Oficyna Wydawnicza PW, Warszawa, 1996 (in Polish).

[10] J. Martinet, Singularities of Smooth Functions and Maps, Cambridge University Press, Cambridge, 1982.

[11] C. T. C. Wall, Finite determinacy of smooth map germs, Bull. London Math. Soc. 13 (1981), 481-539. 
